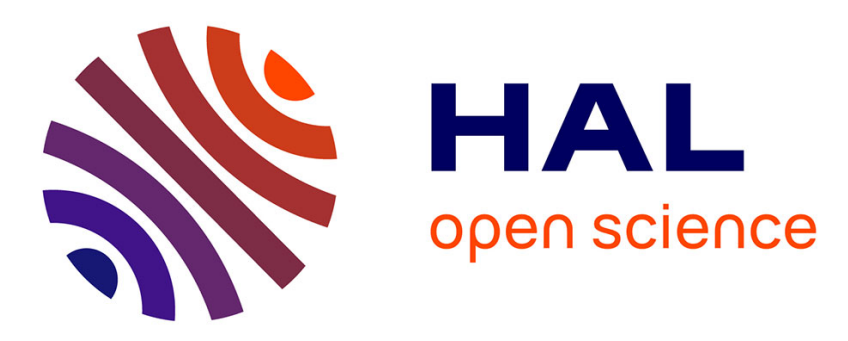

\title{
Modeling of smart materials with thermal effects: dynamic and quasi-static evolution
}

\author{
Francesco Bonaldi, Giuseppe Geymonat, Françoise Krasucki
}

\section{To cite this version:}

Francesco Bonaldi, Giuseppe Geymonat, Françoise Krasucki. Modeling of smart materials with thermal effects: dynamic and quasi-static evolution. Mathematical Models and Methods in Applied Sciences, 2015, 25 (14), pp.2633-2667. 10.1142/S0218202515500578 . hal-01167897

\section{HAL Id: hal-01167897 \\ https://hal.science/hal-01167897}

Submitted on 24 Jun 2015

HAL is a multi-disciplinary open access archive for the deposit and dissemination of scientific research documents, whether they are published or not. The documents may come from teaching and research institutions in France or abroad, or from public or private research centers.
L'archive ouverte pluridisciplinaire HAL, est destinée au dépôt et à la diffusion de documents scientifiques de niveau recherche, publiés ou non, émanant des établissements d'enseignement et de recherche français ou étrangers, des laboratoires publics ou privés. 


\title{
MODELING OF SMART MATERIALS WITH THERMAL EFFECTS: DYNAMIC AND QUASI-STATIC EVOLUTION
}

\author{
FRANCESCO BONALDI \\ Institut Montpelliérain Alexander Grothendieck, UMR-CNRS 5149, \\ Université de Montpellier, Place Eugène Bataillon, 34095 Montpellier Cedex 5, France \\ francesco.bonaldi@univ-montp2.fr \\ GIUSEPPE GEYMONAT \\ Laboratoire de Mécanique des Solides, UMR-CNRS 7649, \\ École Polytechnique, Route de Saclay, 91128 Palaiseau Cedex, France \\ giuseppe.geymonat@lms.polytechnique.fr \\ FRANÇOISE KRASUCKI \\ Institut Montpelliérain Alexander Grothendieck, UMR-CNRS 5149, \\ Université de Montpellier, Place Eugène Bataillon, 34095 Montpellier Cedex 5, France \\ francoise.krasucki@univ-montp2.fr
}

\begin{abstract}
We present a mathematical model for linear magneto-electro-thermo-elastic continua, as sensors and actuators can be thought of, and prove the well-posedness of the dynamic and quasi-static problems. The two proofs are accomplished, respectively, by means of the Hille-Yosida theory and of the Faedo-Galerkin method. A validation of the quasi-static hypothesis is provided by a nondimensionalization of the dynamic problem equations. We also hint at the study of the convergence of the solution to the dynamic problem to that to the quasi-static problem as a small parameter - the ratio of the largest propagation speed for an elastic wave in the body to the speed of light - tends to zero.
\end{abstract}

Keywords: piezoelectricity; magnetostriction; pyroelectricity; pyromagnetism; smart structures; sensors; actuators; nondimensionalization; multiscale problems; semigroups.

\section{Introduction}

When an elastic structure is subjected to a system of external loads, it undergoes a passive deformation. In the case of the so-called smart structures, the strain state is constantly under control by means of sensors and actuators, usually made of piezoelectric and/or magnetostrictive materials and integrated within the structure. In this paper, we consider magneto-electro-thermo-elastic materials as smart materials. The mechanical coupling of piezoelectric and magnetostrictive components in such structures gives rise to the so-called magnetoelectric effect, which is not present in the individual components. Typical geometries where such an effect may take place are given, for instance, by multilayer composites [20,21], by structures made up of a homogeneous matrix within which particles of various form (mostly ellipsoidal) are dispersed [12,13], or even fibrous materials, where parallel cylinders 
are inserted into the homogeneous matrix [1]. For a detailed description of the couplings and the multiphysical phenomenologies occurring in such structures, as well as of their applications, see, e.g., references [2], [9], [16], [17], [23] and [26]. In most literature, the coupling between mechanical and magnetic effects is expressed by a linear constitutive equation, and thus the word 'magnetostrictive' is replaced by piezomagnetic; we will adopt the same point of view and convention in the sequel.

The goal of this work is to enrich the classic models of piezoelectric and piezomagnetic sensors, by taking account of the temperature influence, which in some cases cannot be neglected; for instance, the effects of pyroelectricity and pyromagnetism may be relevant for what concerns energy harvesting performances [20]. To this purpose, it is necessary to add one further equation to the model, i.e., the energy balance. A distinctive feature of the problems encountered in applications is the presence of several parameters, which show the coexistence of different scales: for instance, the thickness of the piezoelectric/piezomagnetic layer may be small with respect to the other dimensions of the structure, the temperature influence may be relevant only on certain unknowns or on certain parts of the multi-structure, etc. In most situations, the superimposition of two wave propagation phenomena characterized by completely different velocities, as is the case with elastic and electromagnetic waves, entails an unworkable numerical treatment of the problem. This issue can be addressed by resorting to a quasi-static model, where the expression 'quasi-static' refers to the assumption that the electric and magnetic fields can be expressed as gradients of the corresponding potentials. This assumption is justified by means of a nondimensionalization procedure, carried out on the equations of the problem, which points out the influence of a small parameter $\delta$ - namely, the ratio between the largest propagation speed for an elastic wave in the body and the speed of light. Such a procedure was performed in [18] for a piezoelectric material without considering the temperature effects; an a priori quasi-static assumption was made, e.g., in [4] and [25] in the case of a thermo-piezoelectric material, whereas the same hypothesis for a magneto-electro-thermo-elastic material was made in [1] and [3].

In this paper, after discussing modeling aspects, attention is focused on the well-posedness of the problem in its most general setting - referred to as dynamic problem in the sequel - whose proof is accomplished by virtue of the Hille-Yosida theory (Section 1). In Section 2, a formal nondimensionalization of the equations is performed, so as $(i)$ to extend the results by [18] and (ii) to justify the quasi-static assumption of [1], [3], [4] and [25]; then, the well-posedness of the quasi-static problem is obtained by virtue of the Faedo-Galerkin method, along the lines of Lions and Miara [24,25]. Finally, we provide another justification of the convergence of the solution to the dynamic problem to that of the quasi-static problem as $\delta \rightarrow 0$. We conclude with a discussion about the rigorous mathematical justification of this convergence and an overview about addressed and unaddressed problems related to the mathematical modeling of smart materials. Typical numerical values of the material parameters involved in the problem are listed in Table 1 of the Appendix. These 
values have been obtained from [22] taking into account the corrections pointed out by [14], [20] and [32] and adding an estimation of the calorific capacity from [21] and of the thermal conductivity from [27].

\section{Notation}

In what follows, we always assume a cartesian frame to have been fixed once and for all in the usual three-dimensional euclidean point space. Thus, we identify with (and denote by) $\mathbb{R}^{3}$ both this space and its associated translation vector space. Throughout the paper, $\Omega \subset \mathbb{R}^{3}$ denotes an open, bounded, connected region, with Lipschitz-continuous boundary $\partial \Omega$, occupied by a continuum made of magnetoelectro-thermo-elastic material in its reference configuration. The typical point of $\Omega$ is denoted by $x$ and time by $t$. A function and its typical value are denoted by the same letter. Time derivative of (scalar, vector or tensor) field $\Phi$ is denoted by either $\dot{\Phi}$ or $\partial_{t} \Phi^{\text {a }}$. Scalars are denoted by light-face letters, vector and tensor fields of any order by bold-face letters. The word 'tensor' is used as a synonym of 'linear transformation between vector spaces', as is customary in continuum mechanics. Unless noted otherwise, the matrix representation of tensor $\mathbf{A}$ (with respect to the fixed cartesian base) is denoted by $[\mathbf{A}]$. The scalar product of tensors $\mathbf{A}$ and $\mathbf{B}$ is denoted ${ }^{\mathrm{b}}$ by $\mathbf{A}: \mathbf{B}$, of vectors $\mathbf{a}$ and $\mathbf{b}$ by $\mathbf{a} \cdot \mathbf{b}$, the cross product by $\mathbf{a} \times \mathbf{b}$, and the euclidean norm of $\mathbf{a}$ by $|\mathbf{a}|$. The symmetric part of second-order tensor $\mathbf{A}$ is denoted by $\operatorname{sym} \mathbf{A}$, the linear space of symmetric second-order tensors by Sym. At times, we also make use of Einstein's summation convention, whereby the summation symbol is suppressed and summation over all possible values of an index is signaled implicitly by the fact that it occurs twice in a monomial term. The following notations are also used:

$$
\begin{array}{ll}
\mathbf{L}^{2}(\Omega):=\left[L^{2}(\Omega)\right]^{k} \text { for } k=3,6, & \mathbf{H}^{1}(\Omega):=\left[H^{1}(\Omega)\right]^{3}, \\
\mathbf{L}^{2}(\Gamma):=\left[L^{2}(\Gamma)\right]^{3} \text { for } \Gamma \subset \partial \Omega, & \mathbf{H}(\operatorname{curl}, \Omega):=\left\{\mathbf{v} \in \mathbf{L}^{2}(\Omega): \nabla \times \mathbf{v} \in \mathbf{L}^{2}(\Omega)\right\}
\end{array}
$$

where $H^{1}(\Omega)$ is the usual Hilbert-Sobolev space ${ }^{\mathrm{c}}$.

\section{Dynamic Problem}

The system of field equations we resort to consists of the point-wise momentum balance equation for three-dimensional continua, Maxwell's equations and the energy balance equation, in its version adapted to deformable electromagnetic materials

\footnotetext{
${ }^{a}$ All three denote a partial derivative, as all formulations hold in a fixed reference domain. ${ }^{\mathrm{b}}$ By definition, $\mathbf{A}: \mathbf{B}:=\operatorname{tr}\left(\mathbf{A B}^{T}\right)=\sum_{i, j=1}^{3} A_{i j} B_{i j}$ for $\mathbf{A}$ and $\mathbf{B}$ arbitrary second-order tensors. ${ }^{\mathrm{c}}$ See [6] and, in particular, [7] and [15] for a detailed description of $\mathbf{H}(\operatorname{curl}, \Omega)$.
} 
(see, e.g., [10] for the case of a thermo-piezoelectric material):

$$
\begin{cases}\rho \ddot{\mathbf{u}}-\operatorname{div} \boldsymbol{\sigma}=\mathbf{f} & x \in \Omega, t>0, \\ \operatorname{div} \mathbf{D}=\rho_{e} & x \in \Omega, t>0, \\ \operatorname{div} \mathbf{B}=0 & x \in \Omega, t>0, \\ \dot{\mathbf{D}}-\nabla \times \mathbf{H}=-\mathbf{J} & x \in \Omega, t>0, \\ \dot{\mathbf{B}}+\nabla \times \mathbf{E}=\mathbf{0} & x \in \Omega, t>0, \\ \dot{\epsilon}+\operatorname{div} \mathbf{q}-\boldsymbol{\sigma}: \dot{\mathbf{e}}-\dot{\mathbf{D}} \cdot \mathbf{E}-\dot{\mathbf{B}} \cdot \mathbf{H}=h & x \in \Omega, t>0,\end{cases}
$$

where $\rho$ is the mass density, $\rho_{e}$ the free electric charge volume density, $\boldsymbol{\sigma}$ the Cauchy stress tensor, $\mathbf{D}$ the electric displacement, $\mathbf{B}$ the magnetic induction, $\epsilon$ the internal energy per unit volume, $\mathbf{q}$ the heat influx, $\mathbf{f}$ the body force, $\mathbf{J}$ an external current density, $h$ an external heat supply, $\mathbf{e}=\mathbf{e}(\mathbf{u})=$ sym $\nabla \mathbf{u}$ the strain tensor, $\mathbf{u}$ the displacement field, $\mathbf{E}$ the electric field and $\mathbf{H}$ the magnetic field. Equations $(1.1)_{2}$ to $(1.1)_{5}$ are usually referred to as, respectively, Gauss's law, Gauss's law for magnetism, Ampère's circuital law and Faraday's law of induction. We explicitly remark that $\rho_{e}$ and $\mathbf{J}$ obey the following continuity equation (conservation of electric charge):

$$
\dot{\rho}_{e}+\operatorname{div} \mathbf{J}=0, \quad x \in \Omega, t>0 .
$$

Boundary and initial conditions will be detailed later.

\subsection{Constitutive Assumptions}

The model presented here is formulated in terms of four unknowns: the displacement field $\mathbf{u}$, the electric field $\mathbf{E}$, the magnetic field $\mathbf{H}$ and the absolute temperature $T$. However, in most situations, it is more convenient to replace $T$ by the temperature variation $\theta$ with respect to a reference value $T_{0}$. In this section we introduce the linear coupled constitutive equations relating the set of state quantities $(\mathbf{e}, \mathbf{E}, \mathbf{H}, \theta)$, with $\mathbf{e}=\operatorname{sym} \nabla \mathbf{u}$, to the corresponding set of dual quantities $(\boldsymbol{\sigma}, \mathbf{D}, \mathbf{B}, s)$ where $s$ is the entropy per unit volume, and show that these equations are consistent with continuum thermodynamics (see, e.g., [8] or [10]).

The point-wise version of the Second Principle of Thermodynamics (entropy imbalance) reads

$$
\dot{s} \geq-\operatorname{div}\left(T^{-1} \mathbf{q}\right)+T^{-1} h, \quad x \in \Omega, t>0,
$$

with $T>0$ the absolute temperature. Upon introducing the electromagnetic enthalpy

$$
G:=\epsilon-T s-\mathbf{D} \cdot \mathbf{E}-\mathbf{B} \cdot \mathbf{H}
$$

it is easy to arrive at the following version of the entropy imbalance, which does not involve the heat supply $h$ :

$$
\dot{G}+s \dot{T}-\boldsymbol{\sigma}: \dot{\mathbf{e}}+\mathbf{D} \cdot \dot{\mathbf{E}}+\mathbf{B} \cdot \dot{\mathbf{H}}+T^{-1} \mathbf{q} \cdot \nabla T \leq 0 ;
$$


for this reason, (1.5) is often referred to as reduced dissipation inequality. The inequality suggests that the quantities in the need of constitutive specifications are electromagnetic enthalpy, entropy, stress, electric displacement, magnetic induction and heat influx. We make the following constitutive assumptions:

$$
\begin{array}{lll}
G=G(\mathbf{e}, \mathbf{E}, \mathbf{H}, T, \nabla T), & s=s(\mathbf{e}, \mathbf{E}, \mathbf{H}, T, \nabla T), & \mathbf{q}=\mathbf{q}(\mathbf{e}, \mathbf{E}, \mathbf{H}, T, \nabla T), \\
\boldsymbol{\sigma}=\boldsymbol{\sigma}(\mathbf{e}, \mathbf{E}, \mathbf{H}, T, \nabla T), & \mathbf{D}=\mathbf{D}(\mathbf{e}, \mathbf{E}, \mathbf{H}, T, \nabla T), \quad \mathbf{B}=\mathbf{B}(\mathbf{e}, \mathbf{E}, \mathbf{H}, T, \nabla T),
\end{array}
$$

with which (1.5) takes the form

$$
\begin{aligned}
\partial_{\nabla T} G \cdot \nabla \dot{T}+\left(\partial_{\mathbf{e}} G-\boldsymbol{\sigma}\right): \dot{\mathbf{e}} & +\left(\partial_{T} G+s\right) \dot{T}+\left(\partial_{\mathbf{E}} G+\mathbf{D}\right) \cdot \dot{\mathbf{E}}+ \\
& +\left(\partial_{\mathbf{H}} G+\mathbf{B}\right) \cdot \dot{\mathbf{H}}+T^{-1} \mathbf{q} \cdot \nabla T \leq 0
\end{aligned}
$$

and require, as in [11], that (1.6) be satisfied whatever the local continuation of any conceivable process; that is, on defining the state $\Theta:=(\mathbf{e}, \mathbf{E}, \mathbf{H}, T, \nabla T) \in$ Sym $\times \mathbb{R}^{3} \times \mathbb{R}^{3} \times \mathbb{R} \times \mathbb{R}^{3},(1.6)$ must hold whatever $\dot{\Theta}$ at whatever state $\Theta$. It follows that:

$$
\partial_{\nabla T} G=\mathbf{0} \Longleftrightarrow G=G(\mathbf{e}, \mathbf{E}, \mathbf{H}, T),
$$

$$
\begin{gathered}
s(\mathbf{e}, \mathbf{E}, \mathbf{H}, T)=-\partial_{T} G(\mathbf{e}, \mathbf{E}, \mathbf{H}, T), \quad \boldsymbol{\sigma}(\mathbf{e}, \mathbf{E}, \mathbf{H}, T)=\partial_{\mathbf{e}} G(\mathbf{e}, \mathbf{E}, \mathbf{H}, T), \\
\mathbf{D}(\mathbf{e}, \mathbf{E}, \mathbf{H}, T)=-\partial_{\mathbf{E}} G(\mathbf{e}, \mathbf{E}, \mathbf{H}, T), \quad \mathbf{B}(\mathbf{e}, \mathbf{E}, \mathbf{H}, T)=-\partial_{\mathbf{H}} G(\mathbf{e}, \mathbf{E}, \mathbf{H}, T), \\
\mathbf{q}(\mathbf{e}, \mathbf{E}, \mathbf{H}, T, \nabla T) \cdot \nabla T \leq 0 .
\end{gathered}
$$

We have now to assign the expression of $G$ in terms of the state quantities. With a view toward getting linear constitutive equations, we introduce the temperature variation $\theta$, by writing

$$
\begin{gathered}
T(x, t)=T_{0}+\theta(x, t), \quad x \in \Omega, t>0, \\
\sup _{x \in \Omega} \theta(x, t) / T_{0} \ll 1 \quad \forall t>0,
\end{gathered}
$$

with $T_{0}>0$ the (constant) reference temperature of the body; we replace $T$ by $\theta$ in the list of state quantities and choose ${ }^{\mathrm{d}}$

$$
\begin{aligned}
G=G(\mathbf{e}, \mathbf{E}, \mathbf{H}, \theta)= & \left(\frac{1}{2} \mathbf{C e}-\mathbf{P}^{T} \mathbf{E}-\mathbf{R}^{T} \mathbf{H}-\boldsymbol{\beta} \theta\right): \mathbf{e}-\frac{1}{2} \mathbf{X E} \cdot \mathbf{E}-\frac{1}{2} \mathbf{M H} \cdot \mathbf{H}+ \\
& -\boldsymbol{\alpha} \mathbf{E} \cdot \mathbf{H}-(\mathbf{p} \cdot \mathbf{E}+\mathbf{m} \cdot \mathbf{H}) \theta-\frac{1}{2} c_{v} \theta^{2} .
\end{aligned}
$$

Let $\widetilde{\Theta}:=(\mathbf{e}, \mathbf{E}, \mathbf{H}, \theta) \in \operatorname{Sym} \times \mathbb{R}^{3} \times \mathbb{R}^{3} \times \mathbb{R}$. It follows from (1.7) that (see, e.g., [3], $[20],[33])$ :

$$
\begin{aligned}
& \boldsymbol{\sigma}=\boldsymbol{\sigma}(\widetilde{\Theta})=\mathbf{C e}-\mathbf{P}^{T} \mathbf{E}-\mathbf{R}^{T} \mathbf{H}-\boldsymbol{\beta} \theta \\
& \mathbf{D}=\mathbf{D}(\widetilde{\Theta})=\mathbf{P e}+\mathbf{X E}+\boldsymbol{\alpha} \mathbf{H}+\mathbf{p} \theta \\
& \mathbf{B}=\mathbf{B}(\widetilde{\Theta})=\mathbf{R e}+\boldsymbol{\alpha} \mathbf{E}+\mathbf{M H}+\mathbf{m} \theta \\
& s=s(\widetilde{\Theta})=\boldsymbol{\beta}: \mathbf{e}+\mathbf{p} \cdot \mathbf{E}+\mathbf{m} \cdot \mathbf{H}+c_{v} \theta .
\end{aligned}
$$

\footnotetext{
${ }^{\mathrm{d}}$ Physical meaning and hypotheses on the constitutive parameters are set forth hereinafter.
} 
In constitutive equations (1.9), $\mathbf{C}=\left(C_{i j k \ell}\right), \mathbf{P}=\left(P_{i j k}\right), \mathbf{R}=\left(R_{i j k}\right), \mathbf{X}=\left(X_{i j}\right)$, $\mathbf{M}=\left(M_{i j}\right), \boldsymbol{\beta}=\left(\beta_{i j}\right), \boldsymbol{\alpha}=\left(\alpha_{i j}\right), \mathbf{p}=\left(p_{i}\right), \mathbf{m}=\left(m_{i}\right)$ and $c_{v}$ represent, respectively, the elasticity tensor, the piezoelectric tensor, the piezomagnetic tensor, the dielectric permittivity tensor, the magnetic permeability tensor, the thermal stress tensor, the magneto-electric tensor, the pyroelectric vector, the pyromagnetic vector, and the calorific capacity, defined such that $c_{v} T_{0}$ be the specific heat per unit volume of the material. Moreover, it can be shown that, whatever the values of the other state variables, the heat influx is null as long as the temperature gradient is; thus, mimicking the constitutive assumption of classic heat conduction, we can generally represent $\mathbf{q}$ as

$$
\mathbf{q}=\mathbf{q}(\widetilde{\Theta}, \nabla \theta)=-\mathbf{K}(\widetilde{\Theta}, \nabla \theta) \nabla \theta,
$$

with $\mathbf{K}=\mathbf{K}(\widetilde{\Theta}, \nabla \theta)$ a second-order tensor. In order to satisfy $(1.7)_{6}, \mathbf{K}$ must obey the following general condition, for any fixed $\widetilde{\Theta}$ :

$$
\mathbf{K} \nabla \theta \cdot \nabla \theta \geq 0, \quad \forall \nabla \theta \in \mathbb{R}^{3} .
$$

In the sequel, we shall consider $\mathbf{K}$ independent of the state variables. Hence, in addition to constitutive relationships (1.9), we have Fourier's law:

$$
\mathbf{q}=\mathbf{q}(\nabla \theta)=-\mathbf{K} \nabla \theta
$$

In $(1.10), \mathbf{K}=\left(K_{i j}\right)$ is the thermal conductivity tensor.

\subsection{Assumptions on the Material Parameters}

The symmetry and positivity conditions we require to be satisfied by density and constitutive parameters are listed below.

- The density $\rho$ is positive:

$$
\rho>0, \quad \rho \in L^{\infty}(\Omega) .
$$

- The fourth-order elasticity tensor $\mathbf{C}=\left(C_{i j k \ell}\right)$ is symmetric and positive definite:

$$
\begin{aligned}
& C_{i j k \ell}=C_{j i k \ell}=C_{k \ell i j}=C_{i j \ell k}, \quad C_{i j k \ell} \in L^{\infty}(\Omega), \\
& C_{i j k \ell} b_{k \ell} b_{i j} \geq \mathcal{C} \sum_{i, j}\left|b_{i j}\right|^{2}, \quad \text { for all } b_{i j}=b_{j i} \in \mathbb{R}, \mathcal{C}>0 .
\end{aligned}
$$

- The third-order piezoelectric tensor ${ }^{\mathrm{e}} \mathbf{P}=\left(P_{i j k}\right)$ is symmetric with respect to the two last indices:

$$
P_{i j k}=P_{i k j}, \quad P_{i j k} \in L^{\infty}(\Omega) .
$$

'By 'third-order tensor' we mean a linear transformation of the vector space of all second-order tensors into $\mathbb{R}^{3}$. For $\mathbf{P}$ a third-order tensor, its transpose $\mathbf{P}^{T}$ maps $\mathbb{R}^{3}$ onto the space of secondorder tensors. In cartesian components, the following identity holds:

$$
P_{i j k}^{T}=P_{k i j} .
$$


- The third-order piezomagnetic tensor $\mathbf{R}=\left(R_{i j k}\right)$ is symmetric with respect to the two last indices:

$$
R_{i j k}=R_{i k j}, \quad R_{i j k} \in L^{\infty}(\Omega) .
$$

- The second-order dielectric permittivity tensor $\mathbf{X}=\left(X_{i j}\right)$ is symmetric and positive definite:

$$
\begin{aligned}
& X_{i j}=X_{j i}, \quad X_{i j} \in L^{\infty}(\Omega), \\
& X_{i j} a_{j} a_{i} \geq \mathcal{X} \sum_{i}\left|a_{i}\right|^{2}, \quad \text { for all } a_{i} \in \mathbb{R}, \mathcal{X}>0 .
\end{aligned}
$$

- The second-order magnetic permeability tensor $\mathbf{M}=\left(M_{i j}\right)$ is symmetric and positive definite:

$$
\begin{aligned}
& M_{i j}=M_{j i}, \quad M_{i j} \in L^{\infty}(\Omega), \\
& M_{i j} a_{j} a_{i} \geq \mu \sum_{i}\left|a_{i}\right|^{2}, \quad \text { for all } a_{i} \in \mathbb{R}, \mu>0 .
\end{aligned}
$$

- The second-order thermal stress tensor $\boldsymbol{\beta}=\left(\beta_{i j}\right)$ is symmetric:

$$
\beta_{i j}=\beta_{j i}, \quad \beta_{i j} \in L^{\infty}(\Omega) .
$$

- The second-order magneto-electric tensor $\boldsymbol{\alpha}=\left(\alpha_{i j}\right)$ is symmetric:

$$
\alpha_{i j}=\alpha_{j i}, \quad \alpha_{i j} \in L^{\infty}(\Omega) .
$$

- The pyroelectric vector $\mathbf{p}=\left(p_{i}\right)$ is such that

$$
p_{i} \in L^{\infty}(\Omega) \text {. }
$$

- The pyromagnetic vector $\mathbf{m}=\left(m_{i}\right)$ is such that

$$
m_{i} \in L^{\infty}(\Omega) .
$$

- The calorific capacity $c_{v}$ is positive:

$$
c_{v}>0, \quad c_{v} \in L^{\infty}(\Omega) .
$$

- The second-order thermal conductivity tensor $\mathbf{K}=\left(K_{i j}\right)$ is symmetric and positive definite:

$$
\begin{aligned}
& K_{i j}=K_{j i}, \quad K_{i j} \in L^{\infty}(\Omega), \\
& K_{i j} a_{j} a_{i} \geq K \sum_{i}\left|a_{i}\right|^{2}, \text { for all } a_{i} \in \mathbb{R}, K>0 .
\end{aligned}
$$

- The following symmetric matrix, referred to in the sequel as coupling matrix (see [25])

$$
\left[\mathbb{M}^{c}\right]:=\left(\begin{array}{ccc}
{[\mathbf{X}]} & {[\boldsymbol{\alpha}]} & {[\mathbf{p}]} \\
{[\boldsymbol{\alpha}]} & {[\mathbf{M}]} & {[\mathbf{m}]} \\
{[\mathbf{p}]^{T}} & {[\mathbf{m}]^{T}} & c_{v}
\end{array}\right)
$$

is positive definite, i.e., there exists a constant $\mathscr{M}>0$ such that

$$
\left[\mathbb{M}^{c}\right] \mathbf{x} \cdot \mathbf{x} \geq \mathscr{M}|\mathbf{x}|^{2}, \quad \forall \mathbf{x} \in \mathbb{R}^{7} \equiv \mathbb{R}^{3} \times \mathbb{R}^{3} \times \mathbb{R}
$$

or, more explicitly,

$$
\begin{aligned}
& X_{i j} a_{j} a_{i}+M_{i j} b_{j} b_{i}+2 \alpha_{i j} a_{j} b_{i}+2\left(p_{k} a_{k}\right) d+2\left(m_{k} a_{k}\right) d+c_{v} d^{2} \geq \\
& \geq C\left(a_{i} a_{i}+b_{i} b_{i}+d^{2}\right), \text { for all } a_{i}, b_{i}, d \in \mathbb{R} .
\end{aligned}
$$




\subsection{Field and Boundary Equations}

Before making explicit the complete system of governing equations, we make some remarks on the energy balance equation. From (1.4) and (1.7), it follows that

$$
\dot{\epsilon}=\boldsymbol{\sigma}: \dot{\mathbf{e}}+T \dot{s}+\dot{\mathbf{D}} \cdot \mathbf{E}+\dot{\mathbf{B}} \cdot \mathbf{H}
$$

with which the energy balance $(1.1)_{6}$ takes the form

$$
T \dot{s}+\operatorname{div} \mathbf{q}=h ;
$$

by $(1.9)_{4}$ and $(1.10)$, the last equation reads

$$
T\left(c_{v} \dot{\theta}+\boldsymbol{\beta}: \dot{\mathbf{e}}+\mathbf{p} \cdot \dot{\mathbf{E}}+\mathbf{m} \cdot \dot{\mathbf{H}}\right)-\operatorname{div} \mathbf{K} \nabla \theta=h,
$$

where $T=T_{0}+\theta$. The left-hand side of this equation contains nonlinear terms (products of $\theta$ and time derivatives of the unknowns); upon neglecting them and setting $r:=h / T_{0}$, we obtain the following linearized version of the energy balance:

$$
c_{v} \dot{\theta}+\boldsymbol{\beta}: \dot{\mathbf{e}}+\mathbf{p} \cdot \dot{\mathbf{E}}+\mathbf{m} \cdot \dot{\mathbf{H}}-\frac{1}{T_{0}} \operatorname{div} \mathbf{K} \nabla \theta=r .
$$

All in all, by virtue of (1.9) and (1.10), the system of field equations (1.1) in the list of unknowns $\widetilde{\mathcal{U}}:=(\mathbf{u}, \mathbf{E}, \mathbf{H}, \theta)$ becomes $^{\mathrm{f}}$ :

$$
\left\{\begin{array}{lll}
\rho \ddot{\mathbf{u}}-\operatorname{div} \mathbf{C e}(\mathbf{u})+\operatorname{div} \mathbf{P}^{T} \mathbf{E}+\operatorname{div} \mathbf{R}^{T} \mathbf{H}+\operatorname{div} \boldsymbol{\beta} \theta=\mathbf{f} & x \in \Omega, & t>0, \\
\operatorname{div}(\mathbf{P e}(\mathbf{u})+\mathbf{X E}+\boldsymbol{\alpha} \mathbf{H}+\mathbf{p} \theta)=\rho_{e} & x \in \Omega, & t>0, \\
\operatorname{div}(\mathbf{R e}(\mathbf{u})+\boldsymbol{\alpha} \mathbf{E}+\mathbf{M} \mathbf{H}+\mathbf{m} \theta)=0 & x \in \Omega, & t>0 \\
\mathbf{X} \dot{\mathbf{E}}+\mathbf{P e}(\dot{\mathbf{u}})+\boldsymbol{\alpha} \dot{\mathbf{H}}+\mathbf{p} \dot{\theta}-\nabla \times \mathbf{H}=-\mathbf{J} & x \in \Omega, & t>0, \\
\mathbf{M} \dot{\mathbf{H}}+\mathbf{R e}(\dot{\mathbf{u}})+\boldsymbol{\alpha} \dot{\mathbf{E}}+\mathbf{m} \dot{\theta}+\nabla \times \mathbf{E}=\mathbf{0} & x \in \Omega, & t>0 \\
c_{v} \dot{\theta}+\boldsymbol{\beta}: \mathbf{e}(\dot{\mathbf{u}})+\mathbf{p} \cdot \dot{\mathbf{E}}+\mathbf{m} \cdot \dot{\mathbf{H}}-\frac{1}{T_{0}} \operatorname{div} \mathbf{K} \nabla \theta=r & x \in \Omega, \quad t>0
\end{array}\right.
$$

Remark 1.1. Note that (1.19) contains twelve scalar equations in ten scalar unknowns. Nevertheless, as we shall show in subsection 1.4 , equations $(1.19)_{2}$ and $(1.19)_{3}$ actually just play the role of compatibility conditions on initial and boundary data, as well as on the free electric charge density $\rho_{e}$.

This system is equipped with the following initial conditions, for any $x \in \Omega$ :

$$
\begin{gathered}
\mathbf{E}(x, 0)=\mathbf{E}_{0}(x), \mathbf{H}(x, 0)=\mathbf{H}_{0}(x), \\
\mathbf{u}(x, 0)=\mathbf{u}_{0}(x), \dot{\mathbf{u}}(x, 0)=\mathbf{u}_{1}(x), \theta(x, 0)=\theta_{0}(x),
\end{gathered}
$$

and with suitable boundary conditions. In particular, let $t>0$ and let $\mathbf{n}$ be the outer unit normal vector field on $\partial \Omega$. As in [34], we consider four time-independent partitions of $\partial \Omega$ : $\left(\Gamma_{m D}, \Gamma_{m N}\right),\left(\Gamma_{e D}, \Gamma_{e N}\right),\left(\Gamma_{g D}, \Gamma_{g N}\right)$ and $\left(\Gamma_{t D}, \Gamma_{t N}\right)$ with $\Gamma_{m D}$, $\Gamma_{e D} \Gamma_{g D}$ and $\Gamma_{t D}$ of strictly positive surface measure. We assign boundary values

${ }^{\mathrm{f}}$ Henceforth, to point out the unknowns of the problem, we replace the list of state quantities $\widetilde{\Theta}$ by the list of unknowns $\widetilde{\mathcal{U}}$ (and $\nabla \theta$ by $\theta$ ) in the constitutive relationships. 
pertaining to mechanical quantities on $\Gamma_{m D}$ and $\Gamma_{m N}$, to electrical quantities on $\Gamma_{e D}$ and $\Gamma_{e N}$, to magnetic quantities on $\Gamma_{g D}$ and $\Gamma_{g N}$ and to thermal quantities on $\Gamma_{t D}$ and $\Gamma_{t N}$. Namely, we assume that the body is clamped along $\Gamma_{m D}$ and subjected to surface traction $\mathbf{g}$ on $\Gamma_{m N}$. Next, it is subjected to a vanishing temperature variation along $\Gamma_{t D}$ and to heat influx $\varrho$ on $\Gamma_{t N}$. Furthermore, the body is in contact with a perfect conductor on $\Gamma_{e D}$ and with an infinitely permeable medium on $\Gamma_{g D}$, whereas it carries an electric charge surface density $d$ on $\Gamma_{e N}$ and a magnetic charge surface density $b$ on $\Gamma_{g N}$. Hence, for any $t>0$, we have

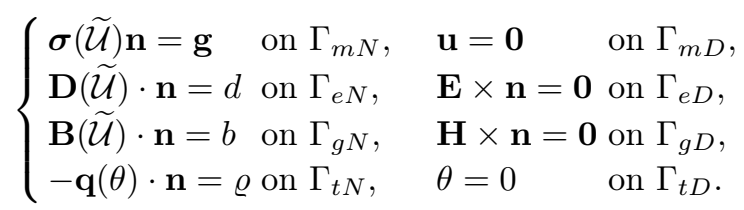

Along with boundary conditions, we introduce the following spaces:

$$
\begin{aligned}
& \mathbf{H}_{m D}^{1}(\Omega):=\left\{\mathbf{v} \in \mathbf{H}^{1}(\Omega): \mathbf{v}=\mathbf{0} \text { on } \Gamma_{m D}\right\}, \\
& H_{t D}^{1}(\Omega):=\left\{v \in H^{1}(\Omega): v=0 \text { on } \Gamma_{t D}\right\},
\end{aligned}
$$

and use analogous definitions for $H_{e D}^{1}(\Omega)$ and $H_{g D}^{1}(\Omega)^{\mathrm{g}}$; finally, let

$$
\mathbf{H}_{e D}(\operatorname{curl}, \Omega):=\left\{\mathbf{v} \in \mathbf{H}(\operatorname{curl}, \Omega): \mathbf{v} \times \mathbf{n}=\mathbf{0} \text { on } \Gamma_{e D}\right\}
$$

and analogously for $\mathbf{H}_{g D}(\operatorname{curl}, \Omega)$ (see, again, [7] and [15]).

\subsection{Existence and Uniqueness}

In order to establish the well-posedness of the problem, we work in the context of the semigroup theory; in particular, we resort to the Hille-Yosida theorem for abstract linear differential equations with a source term [6], whose statement is recalled in the appendix. The main results of this subsection are summarized in the following statement.

Theorem 1.1. Let $T>0$. Assume the initial and boundary values have the following regularity properties:

$$
\begin{gathered}
\left(\mathbf{u}_{0}, \mathbf{u}_{1}, \mathbf{E}_{0}, \mathbf{H}_{0}, \theta_{0}\right) \in \mathbf{H}_{m D}^{1}(\Omega) \times \mathbf{H}_{m D}^{1}(\Omega) \times \mathbf{H}_{e D}(\operatorname{curl}, \Omega) \times \mathbf{H}_{g D}(\operatorname{curl}, \Omega) \times H_{t D}^{1}(\Omega), \\
\operatorname{div}\left(\mathbf{C e}\left(\mathbf{u}_{0}\right)-\mathbf{P}^{T} \mathbf{E}_{0}-\mathbf{R}^{T} \mathbf{H}_{0}-\boldsymbol{\beta} \theta_{0}\right) \in \mathbf{L}^{2}(\Omega), \operatorname{div} \mathbf{K} \nabla \theta_{0} \in L^{2}(\Omega), \\
\mathbf{g} \in C^{3}\left([0, T] ; \mathbf{L}^{2}\left(\Gamma_{m N}\right)\right), \quad \varrho \in C^{3}\left([0, T] ; L^{2}\left(\Gamma_{m N}\right)\right), \\
d(\cdot, t) \in L^{1}\left(\Gamma_{e N}\right) \quad \text { and } \quad b(\cdot, t) \in L^{1}\left(\Gamma_{g N}\right) \quad \forall t \in[0, T] .
\end{gathered}
$$

Also, let the source terms be such that

$$
\begin{aligned}
& \mathbf{f} \in C^{1}\left([0, T] ; \mathbf{L}^{2}(\Omega)\right), \quad \rho_{e} \in H^{1}\left(0, T ; L^{2}(\Omega)\right) \cap C^{0}\left([0, T] ; L^{2}(\Omega)\right), \\
& \mathbf{J} \in C^{1}\left([0, T] ; \mathbf{L}^{2}(\Omega)\right), \quad r \in C^{1}\left([0, T] ; L^{2}(\Omega)\right),
\end{aligned}
$$

gIn particular, spaces $H_{e D}^{1}(\Omega)$ and $H_{g D}^{1}(\Omega)$ will be employed later, in the formulation of the quasi-static problem. 
and let the following compatibility conditions hold:

$$
\begin{aligned}
& \int_{\Gamma_{e N}} d(\cdot, 0) \mathrm{d} \Gamma+\int_{\Gamma_{e D}}\left(\mathbf{P e}\left(\mathbf{u}_{0}\right)+\mathbf{X E}_{0}+\boldsymbol{\alpha} \mathbf{H}_{0}+\mathbf{p} \theta_{0}\right) \cdot \mathbf{n} \mathrm{d} \Gamma=\int_{\Omega} \rho_{e}(\cdot, 0) \mathrm{d} \Omega \\
& \int_{\Gamma_{g N}} b(\cdot, 0) \mathrm{d} \Gamma+\int_{\Gamma_{g D}}\left(\mathbf{R e}\left(\mathbf{u}_{0}\right)+\boldsymbol{\alpha} \mathbf{E}_{0}+\mathbf{M} \mathbf{H}_{0}+\mathbf{m} \theta_{0}\right) \cdot \mathbf{n} \mathrm{d} \Gamma=0 .
\end{aligned}
$$

Finally, let the following electromagnetic duality hypothesis hold:

$$
\Gamma_{g D}=\Gamma_{e N} \quad \text { and } \quad \Gamma_{e D}=\Gamma_{g N} .
$$

Then, problem (1.19)-(1.20)-(1.21) admits a unique strong solution $(\mathbf{u}, \mathbf{E}, \mathbf{H}, \theta)$ satisfying

$$
\left\{\begin{array}{l}
\mathbf{u} \in C^{2}\left([0, T] ; \mathbf{L}^{2}(\Omega)\right) \cap C^{1}\left([0, T] ; \mathbf{H}_{m D}^{1}(\Omega)\right) \\
\mathbf{E} \in C^{1}\left([0, T] ; \mathbf{L}^{2}(\Omega)\right) \cap C^{0}\left([0, T] ; \mathbf{H}_{e D}(\operatorname{curl}, \Omega)\right) \\
\mathbf{H} \in C^{1}\left([0, T] ; \mathbf{L}^{2}(\Omega)\right) \cap C^{0}\left([0, T] ; \mathbf{H}_{g D}(\operatorname{curl}, \Omega)\right) \\
\theta \in C^{1}\left([0, T] ; L^{2}(\Omega)\right) \cap C^{0}\left([0, T] ; H_{t D}^{1}(\Omega)\right)
\end{array}\right.
$$

Proof. The proof is subdivided into six steps.

Step 1. With a view toward applying the Hille-Yosida theorem to our case, we have first of all to reduce our system of field equations into a system of evolution equations. Thus, we rewrite system (1.19) disregarding time-independent Maxwell's equations $(1.19)_{2}$ and $(1.19)_{3}$ and boundary conditions $(1.21)_{2,1}$ and $(1.21)_{3,1}$; we shall come back to these equations later. Therefore, we have

$$
\left\{\begin{array}{lll}
\rho \ddot{\mathbf{u}}-\operatorname{div} \mathbf{C e}(\mathbf{u})+\operatorname{div} \mathbf{P}^{T} \mathbf{E}+\operatorname{div} \mathbf{R}^{T} \mathbf{H}+\operatorname{div} \boldsymbol{\beta} \theta=\mathbf{f} & x \in \Omega, & t>0 \\
\mathbf{X} \dot{\mathbf{E}}+\mathbf{P e}(\dot{\mathbf{u}})+\boldsymbol{\alpha} \dot{\mathbf{H}}+\mathbf{p} \dot{\theta}-\nabla \times \mathbf{H}=-\mathbf{J} & x \in \Omega, & t>0 \\
\mathbf{M} \dot{\mathbf{H}}+\mathbf{R e}(\dot{\mathbf{u}})+\boldsymbol{\alpha} \dot{\mathbf{E}}+\mathbf{m} \dot{\theta}+\nabla \times \mathbf{E}=\mathbf{0} & x \in \Omega, & t>0 \\
c_{v} \dot{\theta}+\boldsymbol{\beta}: \mathbf{e}(\dot{\mathbf{u}})+\mathbf{p} \cdot \dot{\mathbf{E}}+\mathbf{m} \cdot \dot{\mathbf{H}}-\frac{1}{T_{0}} \operatorname{div} \mathbf{K} \nabla \theta=r & x \in \Omega, & t>0
\end{array}\right.
$$

with boundary conditions as in $(1.21)$, except $(1.21)_{2,1}$ and $(1.21)_{3,1}$.

Step 2. As we deal with non-homogeneous boundary conditions, we must introduce trace liftings of the boundary values concerning the displacement field $\mathbf{u}$ and the temperature variation $\theta$. To do so, we resort to the following auxiliary problem actually, to a one-parameter family of static problems, where the parameter is time:

For any $t \geq 0$, find $\widehat{\mathbf{u}}$ and $\widehat{\theta}$ such that ${ }^{\mathrm{h}}$

$$
\begin{cases}\operatorname{div}(\mathbf{C e}(\widehat{\mathbf{u}})-\boldsymbol{\beta} \widehat{\theta})=\mathbf{0}, & x \in \Omega, \\ \boldsymbol{\beta}: \mathbf{e}(\widehat{\mathbf{u}})-\frac{1}{T_{0}} \operatorname{div} \mathbf{K} \nabla \widehat{\theta}=0, & x \in \Omega,\end{cases}
$$

\footnotetext{
${ }^{h}$ Here we write $\widehat{\mathbf{u}}$ and $\widehat{\theta}$ in place of, respectively, $\widehat{\mathbf{u}}(\cdot, t)$ and $\widehat{\theta}(\cdot, t)$ for the sake of notation.
} 
with boundary conditions:

$$
\left\{\begin{array}{llll}
(\mathbf{C e}(\widehat{\mathbf{u}})-\boldsymbol{\beta} \widehat{\theta}) \mathbf{n}=\mathbf{g} & \text { on } \Gamma_{m N}, & \widehat{\mathbf{u}}=\mathbf{0} & \text { on } \Gamma_{m D}, \\
\mathbf{K} \nabla \widehat{\theta} \cdot \mathbf{n}=\varrho & \text { on } \Gamma_{t N}, & \widehat{\theta}=0 & \text { on } \Gamma_{t D} .
\end{array}\right.
$$

Constitutive parameters and boundary values are the same as in (1.19). By resorting to the Lax-Milgram theorem (see [19]) we see that, if boundary values are such that

$$
\mathbf{g} \in C^{3}\left([0, T] ; \mathbf{L}^{2}\left(\Gamma_{m N}\right)\right), \quad \varrho \in C^{3}\left([0, T] ; L^{2}\left(\Gamma_{m N}\right)\right),
$$

then problem (1.25)-(1.26) admits a unique solution $(\widehat{\mathbf{u}}, \widehat{\theta})$ with

$$
\begin{gathered}
\widehat{\mathbf{u}} \in C^{3}\left([0, T] ; \mathbf{H}_{m D}^{1}(\Omega)\right), \\
\widehat{\theta} \in C^{3}\left([0, T] ; H_{t D}^{1}(\Omega)\right) \cap C^{3}\left([0, T] ; H_{\mathbf{K}}^{\Delta}(\Omega)\right),
\end{gathered}
$$

with $H_{\mathbf{K}}^{\Delta}(\Omega):=\left\{\theta \in L^{2}(\Omega): \operatorname{div} \mathbf{K} \nabla \theta \in L^{2}(\Omega)\right\}^{\mathrm{i}}$. On denoting $\widehat{\mathbf{v}}:=\partial_{t} \widehat{\mathbf{u}}$, we have $\left.\widehat{\mathbf{v}}\right|_{\Gamma_{m D}}=\mathbf{0}$. We use functions $\widehat{\mathbf{u}}, \widehat{\mathbf{v}}$ and $\widehat{\theta}$ as trace liftings of boundary values $(1.21)_{1}$ and $(1.21)_{4}$. We shall come back to boundary conditions $(1.21)_{2,1}$ and $(1.21)_{3,1}$ later on.

Step 3. Let $\mathbf{v}:=\partial_{t} \mathbf{u}$ and

$$
\mathbb{H}:=\mathbf{H}_{m D}^{1}(\Omega) \times \mathbf{L}^{2}(\Omega) \times \mathbf{L}^{2}(\Omega) \times \mathbf{L}^{2}(\Omega) \times L^{2}(\Omega) .
$$

Then we can define

$$
U:=\left(\begin{array}{c}
\mathbf{u} \\
\mathbf{v} \\
\mathbf{E} \\
\mathbf{H} \\
\theta
\end{array}\right), \quad U:[0, T] \rightarrow \mathbb{H} .
$$

We also introduce the trace-lifting vector

$$
\widehat{U}:=\left(\begin{array}{c}
\widehat{\mathbf{u}} \\
\widehat{\mathbf{v}} \\
\mathbf{0} \\
\mathbf{0} \\
\widehat{\theta}
\end{array}\right) \quad \text { and } \quad \widetilde{U}:=U-\widehat{U}=\left(\begin{array}{c}
\widetilde{\mathbf{u}} \\
\widetilde{\mathbf{v}} \\
\mathbf{E} \\
\mathbf{H} \\
\widetilde{\theta}
\end{array}\right) \in \mathbb{H} .
$$

We endow the Hilbert space $\mathbb{H}$ with the following scalar product:

$$
\begin{aligned}
\left(U_{1}, U_{2}\right)_{\mathbb{H}}:= & \int_{\Omega} \mathbf{C e}\left(\mathbf{u}_{1}\right): \mathbf{e}\left(\mathbf{u}_{2}\right) \mathrm{d} \Omega+\int_{\Omega} \mathbf{v}_{1} \cdot \mathbf{v}_{2} \mathrm{~d} \Omega+ \\
& +\int_{\Omega} \mathbf{E}_{1} \cdot \mathbf{E}_{2} \mathrm{~d} \Omega+\int_{\Omega} \mathbf{H}_{1} \cdot \mathbf{H}_{2} \mathrm{~d} \Omega+\int_{\Omega} \theta_{1} \theta_{2} \mathrm{~d} \Omega,
\end{aligned}
$$

${ }^{\mathrm{i}}$ That $\widehat{\theta} \in C^{3}\left([0, T] ; H_{\mathbf{K}}^{\Delta}(\Omega)\right)$ follows from the fact that $\widehat{\mathbf{u}} \in C^{3}\left([0, T] ;\left[H_{m D}^{1}(\Omega)\right]^{3}\right)$ and from the second equation of (1.25). 
for all $U_{1}, U_{2} \in \mathbb{H}$. Also, we define the domain $D(\mathcal{A})$ of the differential operator $\mathcal{A}$ which is introduced hereinafter:

$$
\begin{aligned}
D(\mathcal{A}):=\{U \in \mathbb{H}: & \operatorname{div}\left(\mathbf{C e}(\mathbf{u})-\mathbf{P}^{T} \mathbf{E}-\mathbf{R}^{T} \mathbf{H}-\boldsymbol{\beta} \theta\right) \in \mathbf{L}^{2}(\Omega), \mathbf{e}(\mathbf{v}) \in \mathbf{L}^{2}(\Omega), \\
& \nabla \times \mathbf{E} \in \mathbf{L}^{2}(\Omega), \nabla \times \mathbf{H} \in \mathbf{L}^{2}(\Omega), \operatorname{div} \mathbf{K} \nabla \theta \in L^{2}(\Omega), \\
& \left(\mathbf{C e}(\mathbf{u})-\mathbf{P}^{T} \mathbf{E}-\mathbf{R}^{T} \mathbf{H}-\boldsymbol{\beta} \theta\right) \mathbf{n}=\mathbf{0} \text { and } \mathbf{K} \nabla \theta \cdot \mathbf{n}=0 \text { on } \Gamma_{t N}, \\
& \mathbf{u}=\mathbf{0} \text { and } \mathbf{v}=\mathbf{0} \text { on } \Gamma_{m D}, \theta=0 \text { on } \Gamma_{t D}, \\
& \left.\mathbf{E} \times \mathbf{n}=\mathbf{0} \text { on } \Gamma_{e D}, \mathbf{H} \times \mathbf{n}=\mathbf{0} \text { on } \Gamma_{g D}\right\}
\end{aligned}
$$

Step 4. We are now in a position to write system (1.24) in the form of a differential equation:

$$
\begin{gathered}
\mathcal{M} \frac{\mathrm{d} \widetilde{U}}{\mathrm{~d} t}+\mathcal{A} \widetilde{U}=\widetilde{F} \\
\widetilde{F}:=F-\mathcal{A} \widehat{U}-\mathcal{M} \frac{\mathrm{d} \widehat{U}}{\mathrm{~d} t}, \quad F:=\left(\begin{array}{c}
\mathbf{0} \\
\mathbf{f} \\
-\mathbf{J} \\
\mathbf{0} \\
r
\end{array}\right),
\end{gathered}
$$

with

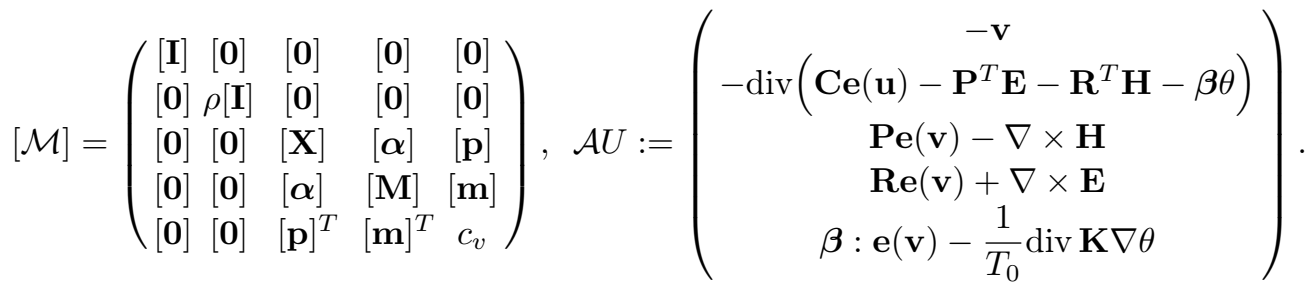

Note that, by $(1.27), \tilde{F} \in C^{1}([0, T] ; \mathbb{H})$ which is in accordance with the hypotheses of the Hille-Yosida theorem. By the hypotheses set forth in subsection $1.2,[\mathcal{M}]$ is a symmetric and positive definite matrix, thus if $\mathcal{M}$ is regarded as an endomorphism of $\mathcal{V}:=\mathbb{R}^{3} \times \mathbb{R}^{3} \times \mathbb{R}^{3} \times \mathbb{R}^{3} \times \mathbb{R}$, it is a symmetric and positive definite linear operator. Therefore, $\mathcal{M}$ admits a unique square root $\mathcal{M}^{1 / 2}$, which is symmetric and positive definite as well. We can then set

$$
\widetilde{V}:=\mathcal{M}^{1 / 2} \widetilde{U}
$$

so as to give (1.30) the form:

$$
\frac{\mathrm{d} \widetilde{V}}{\mathrm{~d} t}+\mathcal{B} \widetilde{V}=\bar{F}, \quad \mathcal{B}:=\mathcal{M}^{-1 / 2} \mathcal{A} \mathcal{M}^{-1 / 2}, \bar{F}:=\mathcal{M}^{-1 / 2} \widetilde{F} .
$$

Let us remark that $\widetilde{V} \in D(\mathcal{B})$ if and only if $\widetilde{U} \in D(\mathcal{A})$, since $\mathcal{B} \widetilde{V}=\mathcal{M}^{-1 / 2} \mathcal{A} \widetilde{U}$ for any $\widetilde{V}=\mathcal{M}^{1 / 2} \widetilde{U}$. Also, it is easy to check that, if $\mathcal{M}$ is symmetric and positive 
definite as an endomorphism of $\mathcal{V}$ with respect to the natural scalar product in $\mathcal{V}$, then $\mathcal{M}$ is self-adjoint and positive definite as a linear operator of $\mathbb{H}$ into itself with respect to scalar product (1.28). Indeed, for any $U_{1}, U_{2} \in \mathbb{H}$, by definition (1.28) it results

$$
\left(\mathcal{M} U_{1}, U_{2}\right)_{\mathbb{H}}=\int_{\Omega} \mathbf{C e}\left(\mathbf{u}_{1}\right): \mathbf{e}\left(\mathbf{u}_{2}\right) \mathrm{d} \Omega+\int_{\Omega} \rho \mathbf{v}_{1} \cdot \mathbf{v}_{2} \mathrm{~d} \Omega+\int_{\Omega} \mathbb{M}^{c} \mathbf{x}_{1} \cdot \mathbf{x}_{2} \mathrm{~d} \Omega,
$$

where in the last term, $\mathbf{x}_{\gamma}:=\left(\mathbf{E}_{\gamma}, \mathbf{H}_{\gamma}, \theta_{\gamma}\right)^{T}, \gamma=1,2$. Now, the last two terms correspond exactly to the submatrix of $[\mathcal{M}]$ obtained by eliminating the first row and the first column, and this submatrix is symmetric and definite positive since $[\mathcal{M}]$ is. By the hypotheses listed in subsection 1.2, $\mathbf{C}$ is symmetric and positive definite as well. Hence we have

$$
\begin{gathered}
\left(\mathcal{M} U_{1}, U_{2}\right)_{\mathbb{H}}=\left(U_{1}, \mathcal{M} U_{2}\right)_{\mathbb{H}}, \quad \forall U_{1}, U_{2} \in \mathbb{H}, \\
(\mathcal{M} U, U)_{\mathbb{H}} \geq \min \left\{\rho_{-}, \mathscr{M}, 1\right\}\|U\|_{\mathbb{H}}^{2}, \forall U \in \mathbb{H},
\end{gathered}
$$

where $\rho_{-}:=\inf _{\Omega} \rho$ and $\mathscr{M}$ denotes the coercivity constant of $\mathbb{M}^{c}$ (see (1.17)).

Step 5. In order to show that the problem

$$
\left\{\begin{array}{l}
\frac{\mathrm{d} \widetilde{V}}{\mathrm{~d} t}+\mathcal{B} \widetilde{V}=\bar{F}, \quad t>0 \\
\widetilde{V}(0)=\widetilde{V}_{0}
\end{array}\right.
$$

is well-posed for any $\widetilde{V}_{0} \in D(\mathcal{B})$, we have to prove that $\mathcal{B}$ is maximal monotone. Note that, since $\mathcal{M}: \mathbb{H} \rightarrow \mathbb{H}$ is a self-adjoint positive definite operator (as well as $\mathcal{M}^{-1 / 2}$ ), we have, for any $\widetilde{V}=\mathcal{M}^{1 / 2} \widetilde{U}$,

$$
(\mathcal{B} \widetilde{V}, \widetilde{V})_{\mathbb{H}}=\left(\mathcal{M}^{-1 / 2} \mathcal{A} \mathcal{M}^{-1 / 2} \widetilde{V}, \widetilde{V}\right)_{\mathbb{H}}=\left(\mathcal{A} \mathcal{M}^{-1 / 2} \widetilde{V}, \mathcal{M}^{-1 / 2} \widetilde{V}\right)_{\mathbb{H}}=(\mathcal{A} \widetilde{U}, \widetilde{U})_{\mathbb{H}} \cdot
$$

Thus, $\mathcal{B}$ is monotone if and only if $\mathcal{A}$ is monotone.

Monotonicity. The proof reduces to showing that $\mathcal{A}$ is monotone. For any $\widetilde{U} \in D(\mathcal{A})$, after integration by parts, we find:

$$
\begin{aligned}
(\mathcal{A} \widetilde{U}, \widetilde{U})_{\mathbb{H}}= & -\int_{\partial \Omega}\left(\mathbf{C e}(\widetilde{\mathbf{u}})-\mathbf{P}^{T} \mathbf{E}-\mathbf{R}^{T} \mathbf{H}-\boldsymbol{\beta} \widetilde{\theta}\right) \mathbf{n} \cdot \widetilde{\mathbf{v}} \mathrm{d} \Gamma-\frac{1}{T_{0}} \int_{\partial \Omega}(\mathbf{K} \nabla \widetilde{\theta} \cdot \mathbf{n}) \widetilde{\theta} \mathrm{d} \Gamma \\
& +\int_{\partial \Omega} \mathbf{E} \times \mathbf{H} \cdot \mathbf{n} \mathrm{d} \Gamma+\frac{1}{T_{0}} \int_{\Omega} \mathbf{K} \nabla \widetilde{\theta} \cdot \nabla \widetilde{\theta} \mathrm{d} \Omega .
\end{aligned}
$$

The first two integrals in the sum vanish, due to homogeneous boundary conditions; also, $1 / T_{0} \int_{\Omega} \mathbf{K} \nabla \widetilde{\theta} \cdot \nabla \widetilde{\theta} \mathrm{d} \Omega \geq 0$ since $\mathbf{K}$ is positive definite, thus:

$$
\begin{aligned}
(\mathcal{A} \widetilde{U}, \widetilde{U})_{\mathbb{H}} & \geq \int_{\partial \Omega} \mathbf{E} \times \mathbf{H} \cdot \mathbf{n} \mathrm{d} \Gamma=\int_{\Gamma_{e D}} \mathbf{E} \times \mathbf{H} \cdot \mathbf{n} \mathrm{d} \Gamma+\int_{\Gamma_{e N}} \mathbf{E} \times \mathbf{H} \cdot \mathbf{n} \mathrm{d} \Gamma= \\
& =\int_{\Gamma_{e D}} \mathbf{E} \times \mathbf{H} \cdot \mathbf{n} \mathrm{d} \Gamma+\int_{\Gamma_{g D}} \mathbf{E} \times \mathbf{H} \cdot \mathbf{n} \mathrm{d} \Gamma,
\end{aligned}
$$


where the last equality holds by assumption (1.23). Taking into account the chain of identities $\mathbf{E} \times \mathbf{H} \cdot \mathbf{n}=-\mathbf{E} \times \mathbf{n} \cdot \mathbf{H}=\mathbf{H} \times \mathbf{n} \cdot \mathbf{E}$ and boundary conditions (1.21) $)_{2,2}$ and $(1.21)_{3,2}$, the right-hand side of the last inequality vanishes as well, hence:

$$
\forall \widetilde{U} \in D(\mathcal{A}), \quad(\mathcal{A} \widetilde{U}, \widetilde{U})_{\mathbb{H}} \geq 0,
$$

i.e., $\mathcal{A}$ is monotone and so is $\mathcal{B}$.

Maximality. The operator $\mathcal{B}+\mathcal{I}$, with $\mathcal{I}$ the identity, is surjective from $D(\mathcal{B})$ into $\mathbb{H}$, i.e., the problem

$$
\mathcal{B} \widetilde{V}+\widetilde{V}=F \quad \text { or, equivalently, } \quad \mathcal{A} \widetilde{U}+\mathcal{M} \widetilde{U}=F
$$

admits a solution $\widetilde{V} \in D(\mathcal{B})(\widetilde{U} \in D(\mathcal{A}))$ for any $F \in \mathbb{H}$. Using the notation $F=\left(\mathbf{F}_{1}, \mathbf{F}_{2}, \mathbf{F}_{3}, \mathbf{F}_{4}, F_{5}\right)$, the first equation of system $\mathcal{A} \widetilde{U}+\mathcal{M} \widetilde{U}=F$ reads

$$
\widetilde{\mathbf{v}}=\widetilde{\mathbf{u}}-\mathbf{F}_{1},
$$

which can be substituted into the other equations to give

$$
\left\{\begin{array}{l}
-\operatorname{div} \mathbf{C e}(\widetilde{\mathbf{u}})+\operatorname{div} \mathbf{P}^{T} \mathbf{E}+\operatorname{div} \mathbf{R}^{T} \mathbf{H}+\operatorname{div} \boldsymbol{\beta} \widetilde{\theta}+\rho \widetilde{\mathbf{u}}=\mathbf{F}_{2}+\rho \mathbf{F}_{1}, \\
\mathbf{P e}(\widetilde{\mathbf{u}})-\nabla \times \mathbf{H}+\mathbf{X E}+\boldsymbol{\alpha} \mathbf{H}+\mathbf{p} \widetilde{\theta}=\mathbf{F}_{3}+\mathbf{P e}\left(\mathbf{F}_{1}\right), \\
\boldsymbol{R e}(\widetilde{\mathbf{u}})+\nabla \times \mathbf{E}+\boldsymbol{\alpha} \mathbf{E}+\mathbf{M H}+\mathbf{m} \widetilde{\theta}=\mathbf{F}_{4}+\mathbf{R e}\left(\mathbf{F}_{1}\right), \\
\boldsymbol{\beta}: \mathbf{e}(\widetilde{\mathbf{u}})-\frac{1}{T_{0}} \operatorname{div} \mathbf{K} \nabla \widetilde{\theta}+\mathbf{p} \cdot \mathbf{E}+\mathbf{m} \cdot \mathbf{H}+c_{v} \widetilde{\theta}=F_{5}+\boldsymbol{\beta}: \mathbf{e}\left(\mathbf{F}_{1}\right) .
\end{array}\right.
$$

Furthermore, $(1.34)_{2}$ allows us to express $\mathbf{E}$ in terms of $\mathbf{u}, \mathbf{H}, \theta$ and source terms $\mathbf{F}_{3}$ and $\mathbf{F}_{1}$; after substituting this expression in the remaining equations, resorting to the weak formulation of the obtained system and applying the Lax-Milgram lemma, we obtain a solution

$$
(\widetilde{\mathbf{u}}, \mathbf{H}, \widetilde{\theta}) \in \mathbf{H}_{m D}^{1}(\Omega) \times \mathbf{H}_{g D}(\operatorname{curl}, \Omega) \times H_{t D}^{1}(\Omega)
$$

for any $F \in \mathbb{H}$, satisfying the homogeneous boundary conditions appearing in the definition of $D(\mathcal{A})$. Of course, by (1.33), $\widetilde{\mathbf{v}} \in \mathbf{H}_{m D}^{1}(\Omega)$ as well; also, one can verify that $\mathbf{E} \in \mathbf{H}_{e D}(\operatorname{curl}, \Omega)$ by taking the curl of the expression giving $\mathbf{E}$ in terms of the other unknowns and using equation $(1.34)_{3}$.

Step 6. By taking the divergence of $(1.24)_{2}$, considering the continuity equation (1.2) and integrating in time, we get $^{j}$

$$
\operatorname{div} \mathbf{D}(x, t)-\operatorname{div} \mathbf{D}(x, 0)=\rho_{e}(x, t)-\rho_{e}(x, 0), \quad x \in \Omega, t>0 .
$$

Now, the point-wise version of hypothesis $(1.22)_{1}$ reads

$$
\operatorname{div} \mathbf{D}(x, 0)=\rho_{e}(x, 0), \quad x \in \Omega,
$$


hence we recover

$$
\operatorname{div} \mathbf{D}(x, t)=\rho_{e}(x, t), \quad x \in \Omega, t>0,
$$

i.e., equation $(1.19)_{2}$. By $(1.22)_{2}$, a completely analogous argument yields

$$
\operatorname{div} \mathbf{B}(x, t)=\operatorname{div} \mathbf{B}(x, 0)=0, \quad x \in \Omega, t>0,
$$

i.e., equation $(1.19)_{3}$. Therefore, equations $(1.19)_{2}$ and $(1.19)_{3}$ are not actually independent of $(1.19)_{4}$ and $(1.19)_{5}$, but they are a consequence of them and of $(1.22)$.

Remark 1.2. The physical meaning of (1.22) is the following: the total volume electric charge must equal the total surface electric charge at $t=0$, and hence at any $t>0$; analogously, the total surface magnetic charge must vanish at $t=0$, and hence at any $t>0$.

\subsection{Energy Functional}

An energy evolution equation satisfied by the solution $(\mathbf{u}, \mathbf{E}, \mathbf{H}, \theta)$ of $(1.24)$ can be formally derived. Indeed, upon defining

$$
\begin{aligned}
\mathcal{E}(t):= & \frac{1}{2} \int_{\Omega} \rho \dot{\mathbf{u}} \cdot \dot{\mathbf{u}} \mathrm{d} \Omega+\frac{1}{2} \int_{\Omega} \mathbf{C e}(\mathbf{u}): \mathbf{e}(\mathbf{u}) \mathrm{d} \Omega+\frac{1}{2} \int_{\Omega} \mathbf{X E} \cdot \mathbf{E} \mathrm{d} \Omega+\frac{1}{2} \int_{\Omega} \mathbf{M H} \cdot \mathbf{H} \mathrm{d} \Omega+ \\
& +\frac{1}{2} \int_{\Omega} c_{v} \theta^{2} \mathrm{~d} \Omega+\int_{\Omega} \boldsymbol{\alpha} \mathbf{E} \cdot \mathbf{H} \mathrm{d} \Omega+\int_{\Omega}(\mathbf{p} \cdot \mathbf{E}) \theta \mathrm{d} \Omega+\int_{\Omega}(\mathbf{m} \cdot \mathbf{H}) \theta \mathrm{d} \Omega,
\end{aligned}
$$

and

$$
L(t):=\int_{\Omega} \mathbf{f} \cdot \dot{\mathbf{u}} \mathrm{d} \Omega-\int_{\Omega} \mathbf{J} \cdot \mathbf{E} \mathrm{d} \Omega+\int_{\Omega} r \theta \mathrm{d} \Omega+\int_{\Gamma_{m N}} \mathbf{g} \cdot \dot{\mathbf{u}} \mathrm{d} \Gamma+\frac{1}{T_{0}} \int_{\Gamma_{t N}} \varrho \theta \mathrm{d} \Gamma,
$$

then by taking the time derivative of $\mathcal{E}$, using equations (1.24) and integrating by parts, we get

$$
\dot{\mathcal{E}}(t)+\frac{1}{T_{0}} \int_{\Omega} \mathbf{K} \nabla \theta \cdot \nabla \theta \mathrm{d} \Omega=L(t), \quad \forall t>0 .
$$

The left-hand side of (1.37) can be thought of as the actual internal power, whereas the right-hand side as the actual external power. The word 'actual' (in place of virtual) is used here to stress the fact that (1.37) is not the variational formulation of system (1.24); indeed, it has been derived considering the solution of (1.24).

Remark 1.3. Note that the free electric charge density $\rho_{e}$, as well as boundary values $b$ and $d$, do not appear in (1.36), since they just intervene in compatibility conditions (1.22). 


\section{Quasi-static Problem}

\subsection{Nondimensionalization of the Equations}

With a view toward justifying the quasi-static hypothesis, i.e., the existence of two scalar fields $\varphi$ and $\zeta$ - respectively, the electric and magnetic potentials - such that

$$
\mathbf{E}=-\nabla \varphi \quad \text { and } \quad \mathbf{H}=-\nabla \zeta,
$$

we nondimensionalize system (1.19) disregarding equations $(1.19)_{2}$ and $(1.19)_{3}$, which do not involve time derivatives of the unknowns; we shall come back to these equations later on. Let $V_{j}(x, \boldsymbol{\nu})$ denote the square root of the $j$-th eigenvalue of the acoustic tensor ${ }^{\mathrm{k}}$ associated with propagation direction $\boldsymbol{\nu}$ and evaluated at $x$, and let

$$
\mathcal{L}:=\sup _{x, y \in \Omega}|x-y|, \quad V_{+}:=\max _{j=1,2,3} \sup _{x \in \Omega} \sup _{|\boldsymbol{\nu}|=1} V_{j}(x, \boldsymbol{\nu}), \quad \mathcal{T}:=\frac{\mathcal{L}}{V_{+}}
$$

be, respectively, the characteristic size of $\Omega$, the maximum propagation speed for an elastic wave in $\Omega$ and the typical time for an elastic wave to travel along distance $\mathcal{L}$. Following Imperiale and Joly [18], we introduce':

$$
\begin{array}{lll}
\rho_{+}:=\sup _{x \in \Omega} \rho(x), & P_{+}:=\sup _{x \in \Omega} \sqrt{\left\|\mathbf{P}(x) \mathbf{P}(x)^{T}\right\|_{2}}, & R_{+}:=\sup _{x \in \Omega} \sqrt{\left\|\mathbf{R}(x) \mathbf{R}(x)^{T}\right\|_{2}}, \\
\alpha_{+}:=\sup _{x \in \Omega}\|\boldsymbol{\alpha}(x)\|_{2}, & p_{+}:=\sup _{x \in \Omega}|\mathbf{p}(x)|, & m_{+}:=\sup _{x \in \Omega}|\mathbf{m}(x)|, \\
c_{v+}:=\sup _{x \in \Omega} c_{v}(x), & \beta_{+}:=\sup _{x \in \Omega}\|\boldsymbol{\beta}(x)\|_{2}, & K_{+}:=\sup _{x \in \Omega}\|\mathbf{K}(x)\|_{2},
\end{array}
$$

and rewrite density and constitutive parameters as:

$$
\begin{array}{lll}
\rho(x)=\rho_{+} \rho_{r}\left(\frac{x}{\mathcal{L}}\right), & \mathbf{C}(x)=\rho_{+} V_{+}^{2} \mathbf{C}_{r}\left(\frac{x}{\mathcal{L}}\right), & \mathbf{P}(x)=P_{+} \mathbf{P}_{r}\left(\frac{x}{\mathcal{L}}\right), \\
\mathbf{R}(x)=R_{+} \mathbf{R}_{r}\left(\frac{x}{\mathcal{L}}\right), \mathbf{X}(x)=\epsilon_{0} \mathbf{X}_{r}\left(\frac{x}{\mathcal{L}}\right), & \mathbf{M}(x)=\mu_{0} \mathbf{M}_{r}\left(\frac{x}{\mathcal{L}}\right), \\
\boldsymbol{\alpha}(x)=\alpha_{+} \boldsymbol{\alpha}_{r}\left(\frac{x}{\mathcal{L}}\right), \mathbf{p}(x)=p_{+} \mathbf{p}_{r}\left(\frac{x}{\mathcal{L}}\right), & \mathbf{m}(x)=m_{+} \mathbf{m}_{r}\left(\frac{x}{\mathcal{L}}\right), \\
c_{v}(x)=c_{v+} c_{v r}\left(\frac{x}{\mathcal{L}}\right), \boldsymbol{\beta}(x)=\beta_{+} \boldsymbol{\beta}_{r}\left(\frac{x}{\mathcal{L}}\right), & \mathbf{K}(x)=K_{+} \mathbf{K}_{r}\left(\frac{x}{\mathcal{L}}\right) .
\end{array}
$$

As for the unknowns, we write:

$$
\begin{array}{ll}
\mathbf{u}(x, t)=\mathcal{L} \mathbf{u}_{r}\left(\frac{x}{\mathcal{L}}, \frac{t}{\mathcal{T}}\right), & \mathbf{E}(x, t)=E^{*} \mathbf{E}_{r}\left(\frac{x}{\mathcal{L}}, \frac{t}{\mathcal{T}}\right), \\
\mathbf{H}(x, t)=H^{*} \mathbf{H}_{r}\left(\frac{x}{\mathcal{L}}, \frac{t}{\mathcal{T}}\right), & \theta(x, t)=T^{*} \theta_{r}\left(\frac{x}{\mathcal{L}}, \frac{t}{\mathcal{T}}\right),
\end{array}
$$

where $\epsilon_{0}$ and $\mu_{0}$ are, respectively, the electric permittivity and magnetic permeability of the vacuum, and $E^{*}, H^{*}$ and $T^{*}$ are to be properly chosen. We recall that the speed of light is given by $c_{0}=\left(\epsilon_{0} \mu_{0}\right)^{-\frac{1}{2}}$.

\footnotetext{
${ }^{\mathrm{k}}$ We recall that the acoustic tensor $\mathbf{A}_{\boldsymbol{\nu}}$ associated with unit vector $\boldsymbol{\nu}$ (the propagation direction), a tensor field over $\Omega$, is the second-order tensor defined by the following condition:

$$
\mathbf{A}_{\nu} \mathbf{a}:=\rho^{-1} \mathbf{C}[\mathbf{a} \otimes \boldsymbol{\nu}] \boldsymbol{\nu}, \quad \forall \mathbf{a} \in \mathbb{R}^{3},
$$

where $\rho$ is the density and $\mathbf{C}$ the elasticity tensor.

${ }^{1}$ In the subsequent definitions, we use the notation $\|\mathbf{A}\|_{2}:=\sqrt{\mathbf{A}: \mathbf{A}}$ for the norm of any secondorder tensor $\mathbf{A}$.
} 
All in all, equations $(1.19)_{1}$ and $(1.19)_{4}$ to $(1.19)_{6}$ become (we still denote by $x$ and $t$ the scaled space and time variables):

$\left\{\begin{array}{l}\rho_{r} \ddot{\mathbf{u}}_{r}-\operatorname{div} \mathbf{C}_{r} \mathbf{e}\left(\mathbf{u}_{r}\right)+\left[\frac{P_{+} E^{*}}{\rho_{+} V_{+}^{2}}\right] \operatorname{div} \mathbf{P}_{r}^{T} \mathbf{E}_{r}+\left[\frac{R_{+} H^{*}}{\rho_{+} V_{+}^{2}}\right] \operatorname{div} \mathbf{R}_{r}^{T} \mathbf{H}_{r}+\left[\frac{\beta_{+} T^{*}}{\rho_{+} V_{+}^{2}}\right] \operatorname{div} \boldsymbol{\beta}_{r} \theta_{r}=\mathbf{f}_{r}, \\ \mathbf{X}_{r} \dot{\mathbf{E}}_{r}+\left[\frac{P_{+}}{\epsilon_{0} E^{*}}\right] \mathbf{P}_{r} \mathbf{e}\left(\dot{\mathbf{u}}_{r}\right)+\left[\frac{\alpha_{+} H^{*}}{\epsilon_{0} E^{*}}\right] \boldsymbol{\alpha}_{r} \dot{\mathbf{H}}_{r}+\left[\frac{p_{+} T^{*}}{\epsilon_{0} E^{*}}\right] \mathbf{p}_{r} \dot{\theta}_{r}-\left[\frac{\sqrt{\mu_{0}} H^{*}}{\sqrt{\epsilon_{0}} E^{*}}\right]\left[\frac{c_{0}}{V_{+}}\right] \nabla \times \mathbf{H}_{r}=-\mathbf{J}_{r}, \\ \mathbf{M}_{r} \dot{\mathbf{H}}_{r}+\left[\frac{R_{+}}{\mu_{0} H^{*}}\right] \mathbf{R}_{r} \mathbf{e}\left(\dot{\mathbf{u}}_{r}\right)+\left[\frac{\alpha_{+} E^{*}}{\mu_{0} H^{*}}\right] \boldsymbol{\alpha}_{r} \dot{\mathbf{E}}_{r}+\left[\frac{m_{+} T^{*}}{\mu_{0} H^{*}}\right] \mathbf{m}_{r} \dot{\theta}_{r}+\left[\frac{\sqrt{\epsilon_{0}} E^{*}}{\sqrt{\mu_{0}} H^{*}}\right]\left[\frac{c_{0}}{V_{+}}\right] \nabla \times \mathbf{E}_{r}=\mathbf{0}, \\ c_{v r} \dot{\theta}_{r}+\left[\frac{\beta_{+}}{c_{v+} T^{*}}\right] \boldsymbol{\beta}_{r}: \mathbf{e}\left(\dot{\mathbf{u}}_{r}\right)+\left[\frac{p_{+} E^{*}}{c_{v} T^{*}}\right]\left(\mathbf{p}_{r} \cdot \dot{\mathbf{E}}_{r}\right)+\left[\frac{m_{+} H^{*}}{c_{v+} T^{*}}\right]\left(\mathbf{m}_{r} \cdot \dot{\mathbf{H}}_{r}\right)-\left[\frac{K_{+}}{V_{+} \mathcal{L} c_{v+} T_{0}}\right] \operatorname{div} \mathbf{K}_{r} \nabla \theta_{r}=r_{r} .\end{array}\right.$

All equations hold for $x \in \widehat{\Omega}$ and $t>0$, with $\widehat{\Omega}:=\{x / \mathcal{L}: x \in \Omega\}$, and all coefficients between square parentheses are dimensionless. We now choose the units of measurement $E^{*}, H^{*}$ and $T^{*}$ in order that the following equalities hold:

$$
\sqrt{\epsilon_{0}} E^{*}=\sqrt{\mu_{0}} H^{*}, \quad \frac{P_{+} E^{*}}{\rho_{+} V_{+}^{2}}=\frac{P_{+}}{\epsilon_{0} E^{*}}, \quad \frac{p_{+} E^{*}}{c_{v+} T^{*}}=\frac{p_{+} T^{*}}{\epsilon_{0} E^{*}} .
$$

This yields

$$
E^{*}=V_{+} \sqrt{\frac{\rho_{+}}{\epsilon_{0}}}, \quad H^{*}=V_{+} \sqrt{\frac{\rho_{+}}{\mu_{0}}}, \quad T^{*}=V_{+} \sqrt{\frac{\rho_{+}}{c_{v+}}} .
$$

Note that this choice of $E^{*}, H^{*}$ and $T^{*}$ ensures symmetry of all other coupling coefficients as well, namely,

$$
\frac{\beta_{+} T^{*}}{\rho_{+} V_{+}^{2}}=\frac{\beta_{+}}{c_{v+} T^{*}}, \quad \frac{R_{+} H^{*}}{\rho_{+} V_{+}^{2}}=\frac{R_{+}}{\mu_{0} H^{*}}, \quad \frac{\alpha_{+} H^{*}}{\epsilon_{0} E^{*}}=\frac{\alpha_{+} E^{*}}{\mu_{0} H^{*}}, \quad \frac{m_{+} T^{*}}{\mu_{0} H^{*}}=\frac{m_{+} H^{*}}{c_{v+} T^{*}} .
$$

Upon setting

$$
\delta:=\frac{V_{+}}{c_{0}},
$$

we can rewrite equations $(2.2)_{2}$ and $(2.2)_{3}$ respectively as follows:

$$
\begin{aligned}
& \nabla \times \mathbf{E}_{r}=-\delta\left(\mathbf{M}_{r} \dot{\mathbf{H}}_{r}+\kappa \mathbf{R}_{r} \mathbf{e}\left(\dot{\mathbf{u}}_{r}\right)+\alpha_{+} c_{0} \boldsymbol{\alpha}_{r} \dot{\mathbf{E}}_{r}+v \mathbf{m}_{r} \dot{\theta}_{r}\right), \\
& \nabla \times \mathbf{H}_{r}=\delta\left(\mathbf{X}_{r} \dot{\mathbf{E}}_{r}+\chi \mathbf{P}_{r} \mathbf{e}\left(\dot{\mathbf{u}}_{r}\right)+\alpha_{+} c_{0} \boldsymbol{\alpha}_{r} \dot{\mathbf{H}}_{r}+\varsigma \mathbf{p}_{r} \dot{\theta}_{r}+\mathbf{J}_{r}\right),
\end{aligned}
$$

with

$$
\kappa:=\frac{R_{+}}{V_{+} \sqrt{\mu_{0} \rho_{+}}}, v:=\frac{m_{+}}{\sqrt{\mu_{0} c_{v+}}}, \chi:=\frac{P_{+}}{V_{+} \sqrt{\epsilon_{0} \rho_{+}}}, \varsigma:=\frac{p_{+}}{\sqrt{\epsilon_{0} c_{v+}}} .
$$

Considering the numerical values of density and elastic moduli for the most classic example of magneto-electro-thermo-elastic composite, i.e., $\mathrm{BaTiO}_{3}-\mathrm{CoFe}_{2} \mathrm{O}_{4}$ with a volume fraction of $\mathrm{BaTiO}_{3}$ equal to 0.6 (see Table 1 in the Appendix), it results

$$
V_{+} \simeq 5980 \mathrm{~m} / \mathrm{s}, \quad \rho_{+}=5600 \mathrm{~kg} / \mathrm{m}^{3},
$$

which give, along with the values of the other constitutive parameters,

$$
\delta \simeq 2 \cdot 10^{-5}, \quad \alpha_{+} c_{0} \simeq 0.75, \quad \kappa \simeq 0.78, \quad v \simeq 0.3, \quad \chi \simeq 9, \quad \varsigma \simeq 2.3 .
$$


Therefore, if the right-hand sides of equations (2.4) remain bounded for any $t>0$, then, in the limit $\delta \rightarrow 0$, we obtain

$$
\nabla \times \mathbf{E}_{r}=\mathbf{0} \text { and } \nabla \times \mathbf{H}_{r}=\mathbf{0}
$$

which means that there exist an electric potential $\varphi_{r}$ and a magnetic potential $\zeta_{r}$ such that

$$
\mathbf{E}_{r}=-\nabla \varphi_{r} \quad \text { and } \quad \mathbf{H}_{r}=-\nabla \zeta_{r}
$$

i.e., the quasi-static hypothesis (2.1).

\subsection{Quasi-static Problem}

As a result, we can remove equations $(1.19)_{4}$ and $(1.19)_{5}$ from system (1.19), introduce the new list of unknowns $\mathcal{U}:=(\mathbf{u}, \varphi, \zeta, \theta)$ and rewrite (1.9)-(1.10) as

$$
\begin{aligned}
& \boldsymbol{\sigma}(\mathcal{U})=\mathbf{C e}(\mathbf{u})+\mathbf{P}^{T} \nabla \varphi+\mathbf{R}^{T} \nabla \zeta-\boldsymbol{\beta} \theta, \\
& \mathbf{D}(\mathcal{U})=\mathbf{P e}(\mathbf{u})-\mathbf{X} \nabla \varphi-\boldsymbol{\alpha} \nabla \zeta+\mathbf{p} \theta, \\
& \mathbf{B}(\mathcal{U})=\mathbf{R e}(\mathbf{u})-\boldsymbol{\alpha} \nabla \varphi-\mathbf{M} \nabla \zeta+\mathbf{m} \theta, \\
& s(\mathcal{U})=\boldsymbol{\beta}: \mathbf{e}(\mathbf{u})-\mathbf{p} \cdot \nabla \varphi-\mathbf{m} \cdot \nabla \zeta+c_{v} \theta, \\
& \mathbf{q}(\theta)=-\mathbf{K} \nabla \theta .
\end{aligned}
$$

Also, (1.19) becomes

$$
\left\{\begin{array}{lll}
\rho \ddot{\mathbf{u}}-\operatorname{div} \boldsymbol{\sigma}(\mathcal{U})=\mathbf{f} & x \in \Omega, & t>0, \\
\operatorname{div} \mathbf{D}(\mathcal{U})=\rho_{e} & x \in \Omega, & t>0, \\
\operatorname{div} \mathbf{B}(\mathcal{U})=0 & x \in \Omega, & t>0, \\
\dot{s}(\mathcal{U})+\frac{1}{T_{0}} \operatorname{div} \mathbf{q}(\theta)=r & x \in \Omega, & t>0,
\end{array}\right.
$$

with initial conditions

$$
\left\{\begin{array}{l}
\mathbf{u}(x, 0)=\mathbf{u}(0)=\mathbf{u}_{0} \text { in } \Omega \\
\dot{\mathbf{u}}(x, 0)=\dot{\mathbf{u}}(0)=\mathbf{u}_{1} \text { in } \Omega \\
\theta(x, 0)=\theta(0)=\theta_{0} \quad \text { in } \Omega
\end{array}\right.
$$

and boundary conditions

$$
\begin{cases}\boldsymbol{\sigma}(\mathcal{U}) \mathbf{n}=\mathbf{g} \quad \text { on } \Gamma_{m N}, & \mathbf{u}=\mathbf{0} \text { on } \Gamma_{m D} \\ \mathbf{D}(\mathcal{U}) \cdot \mathbf{n}=d \text { on } \Gamma_{e N}, & \varphi=0 \text { on } \Gamma_{e D} \\ \mathbf{B}(\mathcal{U}) \cdot \mathbf{n}=b \text { on } \Gamma_{g N}, & \zeta=0 \text { on } \Gamma_{g D} \\ -\mathbf{q}(\theta) \cdot \mathbf{n}=\varrho \text { on } \Gamma_{t N}, & \theta=0 \text { on } \Gamma_{t D}\end{cases}
$$

Let us remark (see [25]) that there is no need for imposing initial conditions on $\varphi$ and $\zeta$, since they are formally given by the unique solution $\left(\varphi_{0}, \zeta_{0}\right)$ of the following 
system, for given $\left(\mathbf{u}_{0}, \theta_{0}\right)^{\mathrm{m}}$

$$
\left\{\begin{array}{l}
\operatorname{div} \mathbf{D}(\mathcal{U}(0))=\operatorname{div}\left(\mathbf{P e}\left(\mathbf{u}_{0}\right)-\mathbf{X} \nabla \varphi_{0}-\boldsymbol{\alpha} \nabla \zeta_{0}+\mathbf{p} \theta_{0}\right)=\rho_{e}(0) \text { in } \Omega \\
\operatorname{div} \mathbf{B}(\mathcal{U}(0))=\operatorname{div}\left(\mathbf{R e}\left(\mathbf{u}_{0}\right)-\boldsymbol{\alpha} \nabla \varphi_{0}-\mathbf{M} \nabla \zeta_{0}+\mathbf{m} \theta_{0}\right)=0 \text { in } \Omega \\
\mathbf{D}(\mathcal{U}(0)) \cdot \mathbf{n}=d(0) \text { on } \Gamma_{e N}, \varphi_{0}=0 \text { on } \Gamma_{e D} \\
\mathbf{B}(\mathcal{U}(0)) \cdot \mathbf{n}=b(0) \text { on } \Gamma_{g N}, \zeta_{0}=0 \text { on } \Gamma_{g D}
\end{array}\right.
$$

\subsection{Energy Functional}

As in the case of problem (1.24), we can define a quasi-static energy

$$
\begin{aligned}
\mathcal{E}_{q s}(t):= & \frac{1}{2} \int_{\Omega} \rho \dot{\mathbf{u}} \cdot \dot{\mathbf{u}} \mathrm{d} \Omega+\frac{1}{2} \int_{\Omega} \mathbf{C e}(\mathbf{u}): \mathbf{e}(\mathbf{u}) \mathrm{d} \Omega+ \\
& +\frac{1}{2} \int_{\Omega} \mathbf{X} \nabla \varphi \cdot \nabla \varphi \mathrm{d} \Omega+\frac{1}{2} \int_{\Omega} \mathbf{M} \nabla \zeta \cdot \nabla \zeta \mathrm{d} \Omega+\frac{1}{2} \int_{\Omega} c_{v} \theta^{2} \mathrm{~d} \Omega+ \\
& +\int_{\Omega} \boldsymbol{\alpha} \nabla \varphi \cdot \nabla \zeta \mathrm{d} \Omega-\int_{\Omega}(\mathbf{p} \cdot \nabla \varphi) \theta \mathrm{d} \Omega-\int_{\Omega}(\mathbf{m} \cdot \nabla \zeta) \theta \mathrm{d} \Omega
\end{aligned}
$$

and a quasi-static external power

$$
\begin{aligned}
L_{q s}(t):= & \int_{\Omega} \mathbf{f} \cdot \dot{\mathbf{u}} \mathrm{d} \Omega+\int_{\Omega} \dot{\rho}_{e} \varphi \mathrm{d} \Omega+\int_{\Omega} r \theta \mathrm{d} \Omega+ \\
& +\int_{\Gamma_{m N}} \mathbf{g} \cdot \dot{\mathbf{u}} \mathrm{d} \Gamma+\frac{1}{T_{0}} \int_{\Gamma_{t N}} \varrho \theta \mathrm{d} \Gamma-\int_{\Gamma_{e N}} \dot{d} \varphi \mathrm{d} \Gamma-\int_{\Gamma_{g N}} \dot{b} \zeta \mathrm{d} \Gamma
\end{aligned}
$$

to get the evolution equation (see [25])

$$
\dot{\mathcal{E}}_{q s}(t)+\frac{1}{T_{0}} \int_{\Omega} \mathbf{K} \nabla \theta \cdot \nabla \theta \mathrm{d} \Omega=L_{q s}(t), \quad \forall t>0 .
$$

Remark 2.1. Note that $\mathcal{E}(t)=\mathcal{E}_{q s}(t)$ for any $t \geq 0$, and that, unlike in the case of (1.37), here the time derivatives of $\rho_{e}, d$ and $b$ are present in the expression of the actual external power on the right-hand side of (2.11).

\subsection{Existence and Uniqueness}

Set $T_{0}=1$ for simplicity and denote by $(\cdot, \cdot)$ the scalar product ${ }^{\mathrm{n}}$ in $L^{2}(\Omega)$ or $\mathbf{L}^{2}(\Omega)$. For any otherfixed $t \in(0, T)$, a weak version of (2.6)-(2.8) takes the following form:

$$
A(\mathcal{U}(t), \mathcal{V})=L(\mathcal{V})
$$

for all $\mathcal{V}=(\mathbf{v}, \psi, \xi, \eta) \in \mathbf{H}_{m D}^{1}(\Omega) \times H_{e D}^{1}(\Omega) \times H_{g D}^{1}(\Omega) \times H_{t D}^{1}(\Omega)$, with

$$
\mathcal{U}(t) \in \mathbf{H}_{m D}^{1}(\Omega) \times H_{e D}^{1}(\Omega) \times H_{g D}^{1}(\Omega) \times H_{t D}^{1}(\Omega),
$$

${ }^{m}$ This statement will be detailed in Remark 2.3 and proven in Lemma 2.1.

${ }^{\mathrm{n}}$ We shall denote by $\|\cdot\|$ the corresponding norm, unless noted otherwise. The scalar product in $L^{2}(\Gamma)$ or $\mathbf{L}^{2}(\Gamma)$, for $\Gamma \subset \partial \Omega$, will be denoted analogously, with a proper subscript. 
where

$$
\begin{aligned}
& A(\mathcal{U}(t), \mathcal{V}):=(\rho \ddot{\mathbf{u}}(t), \mathbf{v})+c(\dot{\mathbf{u}}(t), \eta)+\left(c_{v} \dot{\theta}(t), \eta\right)-d(\dot{\varphi}(t), \eta)-e(\dot{\zeta}(t), \eta)+ \\
&+a_{u}(\mathbf{u}(t), \mathbf{v})+b(\varphi(t), \mathbf{v})-b(\psi, \mathbf{u}(t))+f(\zeta(t), \mathbf{v})-f(\xi, \mathbf{u}(t))+ \\
&-c(\mathbf{v}, \theta(t))+a_{\varphi}(\varphi(t), \psi)+a_{\zeta}(\zeta(t), \xi)+g(\zeta(t), \psi)+g(\varphi(t), \xi)+ \\
&-d(\psi, \theta(t))-e(\xi, \theta(t))+a_{\theta}(\theta(t), \eta), \\
& L(\mathcal{V}):=(\mathbf{f}(t), \mathbf{v})+(r(t), \eta)+\left(\rho_{e}(t), \psi\right)+(\mathbf{g}(t), \mathbf{v})_{\mathbf{L}^{2}\left(\Gamma_{m N}\right)}+ \\
&+(\varrho(t), \eta)_{L^{2}\left(\Gamma_{t N}\right)}-(d(t), \psi)_{L^{2}\left(\Gamma_{e N}\right)}-(b(t), \xi)_{L^{2}\left(\Gamma_{g N}\right)} .
\end{aligned}
$$

where the bilinear forms $a_{u}(\cdot, \cdot), a_{\varphi}(\cdot, \cdot), a_{\zeta}(\cdot, \cdot), a_{\theta}(\cdot, \cdot), b(\cdot, \cdot), c(\cdot, \cdot), d(\cdot, \cdot), e(\cdot, \cdot)$, $f(\cdot, \cdot), g(\cdot, \cdot)$ are defined as follows:

$$
\begin{array}{rlrl}
a_{u}(\mathbf{u}, \mathbf{v}) & :=\int_{\Omega} \mathbf{C e}(\mathbf{u}): \mathbf{e}(\mathbf{v}) \mathrm{d} \Omega, & a_{\varphi}(\varphi, \psi):=\int_{\Omega} \mathbf{X} \nabla \varphi \cdot \nabla \psi \mathrm{d} \Omega, \\
a_{\zeta}(\zeta, \xi) & :=\int_{\Omega} \mathbf{M} \nabla \zeta \cdot \nabla \xi \mathrm{d} \Omega, & & a_{\theta}(\theta, \eta):=\int_{\Omega} \mathbf{K} \nabla \theta \cdot \nabla \eta \mathrm{d} \Omega, \\
b(\psi, \mathbf{u}):=\int_{\Omega} \mathbf{P}^{T} \nabla \psi: \mathbf{e}(\mathbf{u}) \mathrm{d} \Omega, & c(\mathbf{u}, \eta):=\int_{\Omega} \eta \boldsymbol{\beta}: \mathbf{e}(\mathbf{u}) \mathrm{d} \Omega, \\
d(\varphi, \eta):=\int_{\Omega} \eta \mathbf{p} \cdot \nabla \varphi \mathrm{d} \Omega, & e(\zeta, \eta):=\int_{\Omega} \eta \mathbf{m} \cdot \nabla \zeta \mathrm{d} \Omega, \\
f(\xi, \mathbf{u}):=\int_{\Omega} \mathbf{R}^{T} \nabla \xi: \mathbf{e}(\mathbf{u}) \mathrm{d} \Omega, & g(\psi, \zeta):=\int_{\Omega} \boldsymbol{\alpha} \nabla \psi \cdot \nabla \zeta \mathrm{d} \Omega .
\end{array}
$$

Remark 2.2. Note that time derivatives of the unknowns are present in some terms in the definition of $A(\mathcal{U}(t), \mathcal{V})$. We shall give a precise meaning to such terms, as well as to initial conditions (2.7), in the statement of the existence and uniqueness theorem; furthermore we point out that all the terms containing first-order time derivatives are integrated against $\eta \in H_{t D}^{1}(\Omega)$.

Remark 2.3. As noted previously, initial conditions on $\varphi$ and $\zeta$ are given by equations $(2.6)_{2}$ and $(2.6)_{3}$, along with the corresponding boundary conditions. Hence, $\left(\varphi_{0}, \zeta_{0}\right) \in H_{e D}^{1}(\Omega) \times H_{g D}^{1}(\Omega)$ is the unique solution to the following boundary value problem:

$$
\begin{gathered}
a_{\varphi}\left(\varphi_{0}, \psi\right)+a_{\zeta}\left(\zeta_{0}, \xi\right)+g\left(\zeta_{0}, \psi\right)+g\left(\varphi_{0}, \xi\right)=\left(\rho_{e}(0), \psi\right)-(d(0), \psi)_{L^{2}\left(\Gamma_{e N}\right)}+ \\
-(b(0), \xi)_{L^{2}\left(\Gamma_{g N}\right)}+b\left(\psi, \mathbf{u}_{0}\right)+f\left(\xi, \mathbf{u}_{0}\right)+d\left(\psi, \theta_{0}\right)+e\left(\xi, \theta_{0}\right), \\
\forall(\psi, \xi) \in H_{e D}^{1}(\Omega) \times H_{g D}^{1}(\Omega) .
\end{gathered}
$$

Lemma 2.1. Problem (2.15) is well-posed.

Proof. Hypothesis (1.17) implies that the submatrix

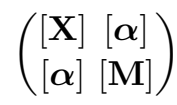


of $\left[\mathbb{M}^{c}\right]$ is symmetric and positive definite. Let $W:=H_{e D}^{1}(\Omega) \times H_{g D}^{1}(\Omega)$. Then, by definitions (2.14), the left-hand side of (2.15) is a continuous and coercive bilinear form (yet symmetric) over $W \times W$, and by the continuity of the trace operator, Poincaré's and Cauchy-Schwarz inequalities, the right-hand side of (2.15) is a bounded linear functional over $W$. The result follows by the Lax-Milgram theorem.

Before giving the statement of well-posedness for the quasi-static problem, we introduce the spaces $\mathbf{H}_{m D}^{*}(\Omega)$ and $H_{t D}^{*}(\Omega)$ as the dual spaces of, respectively, $\mathbf{H}_{m D}^{1}(\Omega)$ and $H_{t D}^{1}(\Omega)$, i.e., the spaces of continuous linear functionals over $\mathbf{H}_{m D}^{1}(\Omega)$ and $H_{t D}^{1}(\Omega)$. As $\mathbf{H}_{m D}^{1}(\Omega)$ and $H_{t D}^{1}(\Omega)$ are dense and continuously embedded in $\mathbf{L}^{2}(\Omega)$ and $L^{2}(\Omega)$ respectively, then we have $[6,28]$

$$
\begin{aligned}
\mathbf{H}_{m D}^{1}(\Omega) & \subset \mathbf{L}^{2}(\Omega) \\
H_{t D}^{1}(\Omega) \subset\left[L^{2}(\Omega)\right. & \simeq\left[L^{2}(\Omega)\right]^{*} \subset \mathbf{H}_{m D}^{*} \subset H_{t D}^{*}(\Omega),
\end{aligned}
$$

where we have used an analogous notation for the dual spaces of $\mathbf{L}^{2}(\Omega)$ and $L^{2}(\Omega)$, and the identification is realized by means of the Riesz-Fréchet representation theorem, being $\mathbf{L}^{2}(\Omega)$ and $L^{2}(\Omega)$ the pivot spaces. In the sequel, we adopt an analogous notation: the linear actions of $\ell \in \mathbf{H}_{m D}^{*}(\Omega)$ or $\ell \in H_{t D}^{*}(\Omega)$ on the corresponding elements $\mathbf{v} \in \mathbf{H}_{m D}^{1}(\Omega)$ and $\eta \in H_{t D}^{1}(\Omega)$ are denoted by $\langle\boldsymbol{\ell}, \mathbf{v}\rangle$ and $\langle\ell, \eta\rangle$, without including subscripts, as meaning will be clear from the context ${ }^{\circ}$.

The results about existence and uniqueness for the quasi-static problem are summarized in the following statement.

Theorem 2.1. Let $T>0$. Assume the following regularity properties on the initial data:

$$
\left(\mathbf{u}_{0}, \mathbf{u}_{1}, \theta_{0}\right) \in \mathbf{H}_{m D}^{1}(\Omega) \times \mathbf{L}^{2}(\Omega) \times H_{t D}^{1}(\Omega),
$$

the following regularity properties on source and boundary values:

$$
\left\{\begin{array}{l}
\mathbf{f} \in L^{2}\left(0, T ; \mathbf{L}^{2}(\Omega)\right), \\
\rho_{e} \in H^{1}\left(0, T ; L^{2}(\Omega)\right) \cap C^{0}\left([0, T] ; L^{2}(\Omega)\right), \\
r \in L^{2}\left(0, T ; L^{2}(\Omega)\right), \\
\mathbf{g} \in H^{2}\left(0, T ; \mathbf{L}^{2}\left(\Gamma_{m N}\right)\right) \cap C^{1}\left([0, T] ; \mathbf{L}^{2}\left(\Gamma_{m N}\right)\right), \\
d \in H^{1}\left(0, T ; L^{2}\left(\Gamma_{e N}\right)\right) \cap C^{0}\left([0, T] ; L^{2}\left(\Gamma_{e N}\right)\right), \\
b \in H^{1}\left(0, T ; L^{2}\left(\Gamma_{g N}\right)\right) \cap C^{0}\left([0, T] ; L^{2}\left(\Gamma_{g N}\right)\right), \\
\varrho \in L^{2}\left(0, T ; L^{2}\left(\Gamma_{t N}\right)\right),
\end{array}\right.
$$

${ }^{\circ}$ Clearly, if $\ell \in \mathbf{L}^{2}(\Omega)$ then $\langle\ell, \mathbf{v}\rangle=(\ell, \mathbf{v}), \forall \mathbf{v} \in \mathbf{H}_{m D}^{1}(\Omega)$ and analogously for $\ell \in L^{2}(\Omega)$ and $v \in H_{t D}^{1}(\Omega)$. 
and the following compatibility conditions:

$$
\begin{cases}\mathbf{g}(0)=\boldsymbol{\sigma}\left(\mathbf{u}_{0}, \varphi_{0}, \zeta_{0}, \theta_{0}\right) \mathbf{n} & \text { on } \Gamma_{m N} \\ d(0)=\mathbf{D}\left(\mathbf{u}_{0}, \varphi_{0}, \zeta_{0}, \theta_{0}\right) \cdot \mathbf{n} & \text { on } \Gamma_{e N} \\ b(0)=\mathbf{B}\left(\mathbf{u}_{0}, \varphi_{0}, \zeta_{0}, \theta_{0}\right) \cdot \mathbf{n} & \text { on } \Gamma_{g N} \\ \varrho(0)=-\mathbf{q}\left(\theta_{0}\right) \cdot \mathbf{n} & \text { on } \Gamma_{t N}\end{cases}
$$

Then, problem (2.12) admits a unique solution $\mathcal{U}=(\mathbf{u}, \varphi, \zeta, \theta)$ such that:

$$
\left\{\begin{array}{l}
\mathbf{u} \in L^{2}\left(0, T ; \mathbf{H}_{m D}^{1}(\Omega)\right) \cap C^{0}\left([0, T] ; \mathbf{L}^{2}(\Omega)\right), \\
\dot{\mathbf{u}} \in L^{2}\left(0, T ; \mathbf{L}^{2}(\Omega)\right), \\
\rho \ddot{\mathbf{u}} \in L^{2}\left(0, T ; \mathbf{H}_{m D}^{*}(\Omega)\right), \\
\varphi \in L^{2}\left(0, T ; H_{e D}^{1}(\Omega)\right), \\
\zeta \in L^{2}\left(0, T ; H_{g D}^{1}(\Omega)\right), \\
\theta \in L^{2}\left(0, T ; H_{t D}^{1}(\Omega)\right), \\
c_{v} \dot{\theta}+\boldsymbol{\beta}: \mathbf{e}(\dot{\mathbf{u}})-\mathbf{p} \cdot \nabla \dot{\varphi}-\mathbf{m} \cdot \nabla \dot{\zeta} \in L^{2}\left(0, T ; H_{t D}^{*}(\Omega)\right) .
\end{array}\right.
$$

Remark 2.4. Based on the solution regularity, the left-hand side terms $(\rho \ddot{\mathbf{u}}(t), \mathbf{v})$ and $\left(c_{v} \dot{\theta}(t), \eta\right)+c(\dot{\mathbf{u}}(t), \eta)-d(\dot{\varphi}(t), \eta)-e(\dot{\zeta}(t), \eta)$ in $(2.12)$ are actually to be thought of as duality pairings.

Proof. We split the proof into six steps.

Step 1. First we consider, as in Step 2 of the proof of Theorem 1.1, the following auxiliary problem for any $t \geq 0$ :

$$
\begin{cases}\operatorname{div} \mathbf{C e}(\widehat{\mathbf{u}})=\mathbf{0}, & x \in \Omega \\ \mathbf{C e}(\widehat{\mathbf{u}}) \mathbf{n}=\mathbf{g} & \text { on } \Gamma_{m N} \\ \widehat{\mathbf{u}}=\mathbf{0} & \text { on } \Gamma_{m D}\end{cases}
$$

Analogously to (1.25)-(1.26), provided

$$
\mathbf{g} \in H^{2}\left(0, T ; \mathbf{L}^{2}\left(\Gamma_{m N}\right)\right) \cap C^{1}\left([0, T] ; \mathbf{L}^{2}\left(\Gamma_{m N}\right)\right),
$$

this problem admits a unique solution

$$
\widehat{\mathbf{u}} \in H^{2}\left(0, T ; \mathbf{H}_{m D}^{1}(\Omega)\right) \cap C^{1}\left([0, T] ; \mathbf{H}_{m D}^{1}(\Omega)\right) .
$$

Let $\overline{\mathbf{u}}:=\mathbf{u}-\widehat{\mathbf{u}}$; we rewrite $(2.12)$ in terms of the list of unknowns $\overline{\mathcal{U}}:=(\overline{\mathbf{u}}, \varphi, \zeta, \theta)$. Then, by (2.13), we have

$$
A(\overline{\mathcal{U}}(t), \mathcal{V})=\bar{L}(\mathcal{V})
$$

with $^{\mathrm{p}}$ 


$$
\begin{aligned}
\bar{L}(\mathcal{V}):= & L(\mathcal{V})-(\mathbf{g}(t), \mathbf{v})_{\mathbf{L}^{2}\left(\Gamma_{m N}\right)}-\left(\rho \partial_{t t} \widehat{\mathbf{u}}(t), \mathbf{v}\right)+ \\
& -(\boldsymbol{\beta}: \mathbf{e}(\widehat{\mathbf{u}}(t)), \eta)+(\mathbf{P e}(\widehat{\mathbf{u}}(t)), \nabla \psi)+(\mathbf{R e}(\widehat{\mathbf{u}}(t)), \nabla \xi) \\
= & (\mathbf{f}(t), \mathbf{v})-\left(\rho \partial_{t t} \widehat{\mathbf{u}}(t), \mathbf{v}\right)+\left(\rho_{e}(t), \psi\right)+(r(t), \eta)-(\boldsymbol{\beta}: \mathbf{e}(\widehat{\mathbf{u}}(t)), \eta)+ \\
& +(\mathbf{P e}(\widehat{\mathbf{u}}(t)), \nabla \psi)+(\operatorname{Re}(\widehat{\mathbf{u}}(t)), \nabla \xi)+ \\
& +(\varrho(t), \eta)_{L^{2}\left(\Gamma_{t N}\right)}-(d(t), \psi)_{L^{2}\left(\Gamma_{e N}\right)}-(b(t), \xi)_{L^{2}\left(\Gamma_{g N}\right)} .
\end{aligned}
$$

Step 2. Let $\left\{\mathbf{v}_{k}\right\}_{k=1}^{\infty}$ and $\left\{\eta_{k}\right\}_{k=1}^{\infty}$ be orthonormal bases for spaces $\mathbf{H}_{m D}^{1}(\Omega)$ and $H_{t D}^{1}(\Omega)$, respectively. Fix $p \in \mathbb{N}$ and define $\mathbf{V}_{p}\left(\Omega, \Gamma_{m D}\right):=\operatorname{span}\left\{\mathbf{v}_{1}, \ldots, \mathbf{v}_{p}\right\}$, $V_{p}\left(\Omega, \Gamma_{t D}\right):=\operatorname{span}\left\{\eta_{1}, \ldots, \eta_{p}\right\}$. These spaces are such that their union for all $p \in \mathbb{N}$ is mutually dense in $\mathbf{H}_{m D}^{1}(\Omega)$ and $H_{t D}^{1}(\Omega)$, respectively. Define

$$
\overline{\mathbf{u}}_{p}(t):=\sum_{k=1}^{p} u_{k}(t) \mathbf{v}_{k}, \quad \theta_{p}(t):=\sum_{k=1}^{p} \vartheta_{k}(t) \eta_{k} .
$$

Furthermore, let $\left(\varphi_{p}(t), \zeta_{p}(t)\right)$ be the unique solution to the following problem:

$$
\begin{aligned}
& \text { Given }\left(\overline{\mathbf{u}}_{p}(t), \theta_{p}(t)\right) \in \mathbf{H}_{m D}^{1}(\Omega) \times H_{t D}^{1}(\Omega), \\
& \text { find }\left(\varphi_{p}(t), \zeta_{p}(t)\right) \in H_{e D}^{1}(\Omega) \times H_{g D}^{1}(\Omega) \text { such that } \\
& a_{\varphi}\left(\varphi_{p}(t), \psi\right)+a_{\zeta}\left(\zeta_{p}(t), \xi\right)+g\left(\zeta_{p}(t), \psi\right)+g\left(\varphi_{p}(t), \xi\right)= \\
& =\left(\rho_{e}(t), \psi\right)-(d(t), \psi)_{L^{2}\left(\Gamma_{e N}\right)}-(b(t), \xi)_{L^{2}\left(\Gamma_{g N}\right)}+b\left(\psi, \overline{\mathbf{u}}_{p}(t)\right)+f\left(\xi, \overline{\mathbf{u}}_{p}(t)\right)+ \\
& +d\left(\psi, \theta_{p}(t)\right)+e\left(\xi, \theta_{p}(t)\right)+(\mathbf{P e}(\widehat{\mathbf{u}}(t)), \nabla \psi)+(\mathbf{R e}(\widehat{\mathbf{u}}(t)), \nabla \xi), \\
& \forall(\psi, \xi) \in H_{e D}^{1}(\Omega) \times H_{g D}^{1}(\Omega) .
\end{aligned}
$$

By (2.18), the sets of coefficients $\left\{u_{k}(t)\right\}_{k=1}^{p}$ and $\left\{\vartheta_{k}(t)\right\}_{k=1}^{p}$ are determined by the solution of the following system of ordinary differential equations:

$$
\left\{\begin{array}{l}
\left\langle\rho \partial_{t t} \overline{\mathbf{u}}_{p}(t), \mathbf{v}_{k}\right\rangle+a_{u}\left(\overline{\mathbf{u}}_{p}(t), \mathbf{v}_{k}\right)+b\left(\varphi_{p}(t), \mathbf{v}_{k}\right)+f\left(\zeta_{p}(t), \mathbf{v}_{k}\right)-c\left(\mathbf{v}_{k}, \theta_{p}(t)\right)= \\
=\left(\mathbf{f}(t), \mathbf{v}_{k}\right)-\left(\rho \partial_{t t} \widehat{\mathbf{u}}(t), \mathbf{v}_{k}\right), \quad \forall k \in\{1, \ldots, p\}, \\
\left\langle c_{v} \dot{\theta}_{p}(t), \eta_{k}\right\rangle+c\left(\partial_{t} \overline{\mathbf{u}}_{p}(t), \eta_{k}\right)-d\left(\dot{\varphi}_{p}(t), \eta_{k}\right)-e\left(\dot{\zeta}_{p}(t), \eta_{k}\right)+a_{\theta}\left(\theta_{p}(t), \eta_{k}\right)= \\
=\left(r(t), \eta_{k}\right)-\left(\boldsymbol{\beta}: \mathbf{e}(\widehat{\mathbf{u}}(t)), \eta_{k}\right)+\left(\varrho(t), \eta_{k}\right)_{L^{2}\left(\Gamma_{t N}\right)}, \quad \forall k \in\{1, \ldots, p\},
\end{array}\right.
$$

with initial conditions such that the following strong convergences hold:

$$
\left\{\begin{array}{l}
\overline{\mathbf{u}}_{p}(0)=\overline{\mathbf{u}}_{p, 0} \rightarrow \overline{\mathbf{u}}_{0} \quad \text { in } \mathbf{H}_{m D}^{1}(\Omega), \\
\partial_{t} \overline{\mathbf{u}}_{p}(0)=\overline{\mathbf{u}}_{p, 1} \rightarrow \overline{\mathbf{u}}_{1} \text { in } \mathbf{L}^{2}(\Omega), \\
\theta_{p}(0)=\theta_{p, 0} \rightarrow \theta_{0} \quad \text { in } H_{t D}^{1}(\Omega),
\end{array}\right.
$$

where $\overline{\mathbf{u}}_{0}:=\overline{\mathbf{u}}(0)=\mathbf{u}(0)-\widehat{\mathbf{u}}(0)=\mathbf{u}_{0}-\widehat{\mathbf{u}}(0), \overline{\mathbf{u}}_{1}:=\partial_{t} \overline{\mathbf{u}}(0)=\dot{\mathbf{u}}(0)-\partial_{t} \widehat{\mathbf{u}}(0)=$ $\mathbf{u}_{1}-\partial_{t} \widehat{\mathbf{u}}(0)$. The linear ordinary differential equations theory guarantees (see [31]) that there exists $t_{p}>0$ such that system (2.21)-(2.22) admits a local solution in time interval $\left[0, t_{p}\right]$. 
Step 3. We rewrite the energy functional associated with the problem in terms of the bilinear forms introduced above:

$$
2 \overline{\mathcal{E}}(t)=\left(\rho \partial_{t} \overline{\mathbf{u}}(t), \partial_{t} \overline{\mathbf{u}}(t)\right)+a_{u}(\overline{\mathbf{u}}(t), \overline{\mathbf{u}}(t))+Q_{\mathbb{M}^{c}}(\varphi(t), \zeta(t), \theta(t)),
$$

where

$$
\begin{gathered}
Q_{\mathbb{M}^{c}}: H_{e D}^{1}(\Omega) \times H_{g D}^{1}(\Omega) \times L^{2}(\Omega) \rightarrow \mathbb{R} \\
Q_{\mathbb{M}^{c}}(\psi, \xi, \eta)=a_{\varphi}(\psi, \psi)+a_{\zeta}(\xi, \xi)+\left(c_{v} \eta, \eta\right)+2 g(\psi, \xi)-2 d(\psi, \eta)-2 e(\xi, \eta)
\end{gathered}
$$

is the quadratic form associated with the coupling matrix. Note that by hypothesis (1.17), there exist a coercivity constant $\mathscr{M}>0$ and a continuity constant $\mathfrak{M}>0$ (depending on the lowest and greatest eigenvalues of $\mathbb{M}^{c}$, respectively) such that

$$
\mathscr{M}\left(\|\nabla \psi\|^{2}+\|\nabla \xi\|^{2}+\|\eta\|^{2}\right) \leq Q_{\mathbb{M}^{c}}(\psi, \xi, \eta) \leq \mathfrak{M}\left(\|\nabla \psi\|^{2}+\|\nabla \xi\|^{2}+\|\eta\|^{2}\right),
$$

for all $(\psi, \xi, \eta) \in H_{e D}^{1}(\Omega) \times H_{g D}^{1}(\Omega) \times L^{2}(\Omega)$.

One can prove that an evolution equation analogous to (2.11) is satisfied by the energy $\overline{\mathcal{E}}_{p}$ associated with $\left(\overline{\mathbf{u}}_{p}, \varphi_{p}, \zeta_{p}, \theta_{p}\right)$, namely,

$$
\partial_{t} \overline{\mathcal{E}}_{p}(t)+a_{\theta}\left(\theta_{p}(t), \theta_{p}(t)\right)=\bar{L}_{p}(t) .
$$

Indeed, first multiply $(2.21)_{1}$ by $u_{k}^{\prime}(t),(2.21)_{2}$ by $\vartheta_{k}^{\prime}(t)$, then make the sum on $k$ ranging from 1 to $p$; finally, take the time derivative of $(2.20)$ and replace $\psi$ by $\varphi_{p}(t)$. By adding the three equations thus obtained, we get (2.25) with

$$
\begin{aligned}
2 \overline{\mathcal{E}}_{p}(t):= & \left(\rho \partial_{t} \overline{\mathbf{u}}_{p}(t), \partial_{t} \overline{\mathbf{u}}_{p}(t)\right)+a_{u}\left(\overline{\mathbf{u}}_{p}(t), \overline{\mathbf{u}}_{p}(t)\right)+Q_{\mathbb{M} c}\left(\varphi_{p}(t), \zeta_{p}(t), \theta_{p}(t)\right), \\
\bar{L}_{p}(t):= & \left(\mathbf{f}(t), \partial_{t} \overline{\mathbf{u}}_{p}(t)\right)-\left(\rho \partial_{t} \widehat{\mathbf{u}}(t), \partial_{t} \overline{\mathbf{u}}_{p}(t)\right)+\left(\dot{\rho}_{e}(t), \varphi_{p}(t)\right)+\left(r(t), \theta_{p}(t)\right)+ \\
& -\left(\boldsymbol{\beta}: \mathbf{e}(\widehat{\mathbf{u}}(t)), \theta_{p}(t)\right)+\left(\mathbf{P e}(\widehat{\mathbf{u}}(t)), \nabla \varphi_{p}(t)\right)+\left(\mathbf{R e}(\widehat{\mathbf{u}}(t)), \nabla \zeta_{p}(t)\right)+ \\
& -\left(\dot{d}(t), \varphi_{p}(t)\right)_{L^{2}\left(\Gamma_{e N}\right)}-\left(\dot{b}(t), \zeta_{p}(t)\right)_{L^{2}\left(\Gamma_{g N}\right)}+\left(\varrho(t), \theta_{p}(t)\right)_{L^{2}\left(\Gamma_{m N}\right) .}
\end{aligned}
$$

Step 4. We shall prove now that the solution $\overline{\mathcal{E}}_{p}$ of $(2.25)$ is bounded for any $t \in\left[0, t_{p}\right]$ by a constant depending on $T$, which allows us to extend the time interval to $[0, T]$. First, we look for a bound on the external power $\bar{L}_{p}(t)$ depending on $\overline{\mathcal{E}}_{p}(t)$. We use the continuity of the trace operator, Poincaré's and Young's inequalities to infer the existence of constants $c_{r, 1}, c_{r, 2}$ and $c_{r, 3}$ such that:

$$
\begin{gathered}
\bar{L}_{p}(t) \leq C_{0}(t)+\frac{1}{2}\left(\left\|\partial_{t} \overline{\mathbf{u}}_{p}(t)\right\|^{2}+\left(c_{r, 1}^{2}+1\right)\left\|\nabla \varphi_{p}(t)\right\|^{2}+\right. \\
\left.+\left(c_{r, 2}^{2}+1\right)\left\|\nabla \zeta_{p}(t)\right\|^{2}+\left\|\theta_{p}(t)\right\|^{2}\right)+\frac{\delta_{0}}{2} c_{r, 3}^{2}\left\|\nabla \theta_{p}(t)\right\|^{2}, \\
2 C_{0}(t):=\|\mathbf{f}(t)\|^{2}+\left\|\rho \partial_{t t} \widehat{\mathbf{u}}(t)\right\|^{2}+\left\|\dot{\rho}_{e}(t)\right\|^{2}+\|\boldsymbol{\beta}: \mathbf{e}(\widehat{\mathbf{u}}(t))\|^{2}+\|\mathbf{P e}(\widehat{\mathbf{u}}(t))\|^{2}+ \\
+\|\mathbf{R e}(\widehat{\mathbf{u}}(t))\|^{2}+\|r(t)\|^{2}+\|\dot{d}(t)\|_{L^{2}\left(\Gamma_{e N}\right)}^{2}+\|\dot{b}(t)\|_{L^{2}\left(\Gamma_{g N}\right)}^{2}+\frac{1}{\delta_{0}}\|\varrho(t)\|_{L^{2}\left(\Gamma_{t N}\right)}^{2} .
\end{gathered}
$$


Let $K>0$ denote the coercivity constant of $a_{\theta}(\cdot, \cdot)$. We select $\delta_{0}$ such that $\widetilde{K}:=K-\frac{\delta_{0}}{2} c_{r, 3}^{2}>0$. By $(2.24)$ we can determine a constant $C>0$ such that $\left(c_{r, 1}^{2}+1\right)\left\|\nabla \varphi_{p}(t)\right\|^{2}+\left(c_{r, 2}^{2}+1\right)\left\|\nabla \zeta_{p}(t)\right\|^{2}+\left\|\theta_{p}(t)\right\|^{2} \leq C Q_{\mathbb{M}^{c}}\left(\varphi_{p}(t), \zeta_{p}(t), \theta_{p}(t)\right) \leq 2 C \overline{\mathcal{E}}_{p}(t)$.

Hence, there exist $C_{0}(t)$ and $C_{1}$ such that

$$
\partial_{t} \overline{\mathcal{E}}_{p}(t)+\widetilde{K}\left\|\nabla \theta_{p}(t)\right\|^{2} \leq L_{p}(t) \leq C_{0}(t)+C_{1} \overline{\mathcal{E}}_{p}(t), \forall t \in\left[0, t_{p}\right]
$$

which gives, upon integrating in time,

$$
\overline{\mathcal{E}}_{p}(t)+\widetilde{K} \int_{0}^{t}\left\|\nabla \theta_{p}(s)\right\|^{2} \mathrm{~d} s \leq \overline{\mathcal{E}}_{p}(0)+\int_{0}^{t}\left(C_{0}(s)+C_{1} \overline{\mathcal{E}}_{p}(s)\right) \mathrm{d} s,
$$

for any $t \in\left[0, t_{p}\right]$. Now, note that $\overline{\mathcal{E}}_{p}(0)$ contains the terms $\left(\rho \partial_{t} \overline{\mathbf{u}}(0), \partial_{t} \overline{\mathbf{u}}(0)\right)$, $a_{u}\left(\overline{\mathbf{u}}_{p}(0), \overline{\mathbf{u}}_{p}(0)\right),\left(c_{v} \theta_{p}(0), \theta_{p}(0)\right)$, which are bounded by $(2.22)$. To bound the remaining terms, we have to find bounds on $\left\|\nabla \varphi_{p}(0)\right\|$ and $\left\|\nabla \zeta_{p}(0)\right\|$. This can be accomplished by taking $t=0$ in (2.20), replacing $\psi$ and $\xi$ by, respectively, $\varphi_{p}(0)$ and $\zeta_{p}(0)$, and then using hypothesis (1.17), Cauchy-Schwarz and Young's inequality, together with the continuity of the trace operator; all in all (we omit details), one finds that there exist a constant $c>0$ depending on the material parameters such that

$$
\begin{aligned}
\left\|\nabla \varphi_{p}(0)\right\|^{2}+\left\|\nabla \zeta_{p}(0)\right\|^{2} \leq & c\left(\left\|\rho_{e}(0)\right\|^{2}+\|d(0)\|_{L^{2}\left(\Gamma_{e N}\right)}^{2}+\|b(0)\|_{L^{2}\left(\Gamma_{g N}\right)}^{2}+\right. \\
& \left.+\|\mathbf{P e}(\widehat{\mathbf{u}}(0))\|^{2}+\|\mathbf{R e}(\widehat{\mathbf{u}}(0))\|^{2}+2\left(\left\|\theta_{p}(0)\right\|^{2}+\left\|\nabla \overline{\mathbf{u}}_{p}(0)\right\|^{2}\right)\right),
\end{aligned}
$$

and since the right-hand side is bounded by $(2.22),\left\|\nabla \varphi_{p}(0)\right\|$ and $\left\|\nabla \zeta_{p}(0)\right\|$ are bounded. Hence, $\overline{\mathcal{E}}_{p}(0)$ is bounded. Moreover, as $C_{0}(t)$ depends only on the data, we can integrate up to $T>0$ and get

$$
\int_{0}^{t} C_{0}(s) \mathrm{d} s \leq \int_{0}^{T} C_{0}(s) \mathrm{d} s=\bar{C}_{0}
$$

$$
\begin{aligned}
2 \bar{C}_{0}:= & \|\mathbf{f}\|_{L^{2}\left(0, T ; \mathbf{L}^{2}(\Omega)\right)}^{2}+\left\|\dot{\rho}_{e}\right\|_{L^{2}\left(0, T ; L^{2}(\Omega)\right)}^{2}+\|r\|_{L^{2}\left(0, T ; L^{2}(\Omega)\right)}^{2}+\left\|\rho \partial_{t t} \widehat{\mathbf{u}}\right\|_{L^{2}\left(0, T ; \mathbf{L}^{2}(\Omega)\right)}^{2}+ \\
& +\|\boldsymbol{\beta}: \mathbf{e}(\widehat{\mathbf{u}})\|_{L^{2}\left(0, T ; L^{2}(\Omega)\right)}^{2}+\|\mathbf{P e}(\widehat{\mathbf{u}})\|_{L^{2}\left(0, T ; \mathbf{L}^{2}(\Omega)\right)}^{2}+\|\mathbf{R e}(\widehat{\mathbf{u}})\|_{L^{2}\left(0, T ; \mathbf{L}^{2}(\Omega)\right)}^{2}+ \\
& +\|\dot{d}\|_{L^{2}\left(0, T ; L^{2}\left(\Gamma_{e N}\right)\right)}^{2}+\|\dot{b}\|_{L^{2}\left(0, T ; L^{2}\left(\Gamma_{g N}\right)\right)}^{2}+\frac{1}{\delta_{0}}\|\varrho\|_{L^{2}\left(0, T ; L^{2}\left(\Gamma_{t N}\right)\right)}^{2} .
\end{aligned}
$$

All in all, we finally obtain the existence of positive constants $\widetilde{K}, k_{1}$ and $k_{2}$ such that

$$
\overline{\mathcal{E}}_{p}(t)+\widetilde{K} \int_{0}^{t}\left\|\nabla \theta_{p}(s)\right\|^{2} \mathrm{~d} s \leq k_{1}+k_{2} \int_{0}^{t} \overline{\mathcal{E}}_{p}(s) \mathrm{d} s, \quad \forall t \in\left[0, t_{p}\right],
$$

whence, by Gronwall's lemma, for any $t \in\left[0, t_{p}\right]$ it results

$$
\overline{\mathcal{E}}_{p}(t) \leq k_{1} e^{k_{2} t} \leq k_{1} e^{k_{2} T}<+\infty, \quad \forall T \in\left[t_{p},+\infty\right) .
$$


Hence, as the solution of $(2.25)$ is uniformly bounded in $\left[0, t_{p}\right]$, we can extend the maximal existence interval to $[0, T]$. As a consequence, we also get

$$
\widetilde{K} \int_{0}^{T}\left\|\nabla \theta_{p}(s)\right\|^{2} \mathrm{~d} s \leq k_{1} e^{k_{2} T} .
$$

Step 5. From the last two bounds, we infer the weak convergence of subsequences still denoted $\left(\overline{\mathbf{u}}_{p}, \varphi_{p}, \zeta_{p}, \theta_{p}\right)$ as follows:

$$
\begin{cases}\overline{\mathbf{u}}_{p} \rightarrow \overline{\mathbf{u}} & \text { in } L^{2}\left(0, T ; \mathbf{H}_{m D}^{1}(\Omega)\right), \\ \partial_{t} \overline{\mathbf{u}}_{p} \rightarrow \partial_{t} \overline{\mathbf{u}} & \text { in } L^{2}\left(0, T ; \mathbf{L}^{2}(\Omega)\right), \\ \varphi_{p} \rightarrow \varphi & \text { in } L^{2}\left(0, T ; H_{e D}^{1}(\Omega)\right), \\ \zeta_{p} \rightarrow \zeta & \text { in } L^{2}\left(0, T ; H_{g D}^{1}(\Omega)\right), \\ \theta_{p} \rightarrow \theta & \text { in } L^{2}\left(0, T ; H_{t D}^{1}(\Omega)\right) .\end{cases}
$$

Moreover, to get an estimate on $\rho \partial_{t t} \overline{\mathbf{u}}_{p}$, we select $\mathbf{v} \in \mathbf{H}_{m D}^{1}(\Omega)$ such that $\|\mathbf{v}\|_{\mathbf{H}_{m D}^{1}(\Omega)} \leq 1$; the projection theorem for separable Hilbert spaces allows us to write $\mathbf{v}=\mathbf{v}^{1}+\mathbf{v}^{2}$, with $\mathbf{v}^{1} \in \operatorname{span}\left\{\mathbf{v}_{k}\right\}_{k=1}^{p}$ and $\left(\mathbf{v}^{2}, \mathbf{v}_{k}\right)_{\mathbf{H}_{m D}^{1}(\Omega)}=0$ for $k=1, \ldots, p$. Then, (2.21) 1 implies that

$$
\begin{aligned}
\left\langle\rho \partial_{t t} \overline{\mathbf{u}}_{p}(t), \mathbf{v}\right\rangle=\left\langle\rho \partial_{t t} \overline{\mathbf{u}}_{p}(t), \mathbf{v}^{1}\right\rangle= & -a_{u}\left(\overline{\mathbf{u}}_{p}(t), \mathbf{v}^{1}\right)-b\left(\varphi_{p}(t), \mathbf{v}^{1}\right)-f\left(\zeta_{p}(t), \mathbf{v}^{1}\right)+ \\
& +c\left(\mathbf{v}^{1}, \theta_{p}(t)\right)+\left(\mathbf{f}(t), \mathbf{v}^{1}\right)-\left(\rho \partial_{t t} \widehat{\mathbf{u}}(t), \mathbf{v}^{1}\right),
\end{aligned}
$$

whence, by using Cauchy-Schwarz and Young's inequalities, integrating in time and taking into account the boundedness of the energy $\mathcal{E}_{p}(t)$, we infer the existence of positive constants $k_{3}$ and $k_{4}$ depending on the material parameters such that

$\left\|\rho \partial_{t t} \overline{\mathbf{u}}_{p}\right\|_{L^{2}\left(0, T ; \mathbf{H}_{m D}^{*}(\Omega)\right)} \leq k_{1} k_{3} T e^{k_{2} T}+k_{4}\left(\|\mathbf{f}\|_{L^{2}\left(0, T ; \mathbf{L}^{2}(\Omega)\right)}^{2}+\left\|\rho \partial_{t t} \widehat{\mathbf{u}}\right\|_{L^{2}\left(0, T ; \mathbf{L}^{2}(\Omega)\right)}^{2}\right)$,

therefore $\rho \partial_{t t} \overline{\mathbf{u}}_{p}$ is bounded in $L^{2}\left(0, T ; \mathbf{H}_{m D}^{*}(\Omega)\right)$ and hence it admits a subsequence still denoted $\rho \partial_{t t} \overline{\mathbf{u}}_{p}$ such that

$$
\rho \partial_{t t} \overline{\mathbf{u}}_{p} \rightarrow \rho \partial_{t t} \overline{\mathbf{u}} \quad \text { in } \quad L^{2}\left(0, T ; \mathbf{H}_{m D}^{*}(\Omega)\right) .
$$

Now, let $\Xi_{p}(t):=c_{v} \dot{\theta}_{p}(t)+\boldsymbol{\beta}: \partial_{t} \mathbf{e}(\overline{\mathbf{u}}(t))-\mathbf{p} \cdot \nabla \dot{\varphi}(t)-\mathbf{m} \cdot \nabla \dot{\zeta}(t)$; with an analogous procedure, if we take $\eta \in H_{t D}^{1}(\Omega)$ with $\|\eta\|_{H_{t D}^{1}(\Omega)} \leq 1$ and write $\eta=\eta^{1}+\eta^{2}$, $\eta^{1} \in \operatorname{span}\left\{\eta_{1}, \ldots, \eta_{k}\right\}$ and $\left(\eta^{2}, \eta_{k}\right)_{H_{t D}^{1}(\Omega)}=0$ for $k=1, \ldots, p$, then $(2.21)_{2}$ reads

$\left\langle\Xi_{p}(t), \eta\right\rangle=\left\langle\Xi_{p}(t), \eta^{1}\right\rangle=-a_{\theta}\left(\theta_{p}(t), \eta^{1}\right)+\left(r(t), \eta^{1}\right)+\left(\boldsymbol{\beta}: \mathbf{e}(\widehat{\mathbf{u}}(t)), \eta^{1}\right)+\left(\varrho(t), \eta^{1}\right)_{L^{2}\left(\Gamma_{t N}\right)}$,

whence, by using Cauchy-Schwarz and Young's inequalities, together with the continuity of the trace operator, integrating in time and taking into account (2.27), we can determine two positive constants $k_{5}$ and $k_{6}$ depending on the material parameters such that

$$
\begin{aligned}
\left\|\Xi_{p}\right\|_{L^{2}\left(0, T ; H_{t D}^{*}(\Omega)\right)} \leq & k_{5}\left\|\nabla \theta_{p}\right\|_{L^{2}\left(0, T ; \mathbf{L}^{2}(\Omega)\right)}+k_{6}\left(\|r\|_{L^{2}\left(0, T ; L^{2}(\Omega)\right)}^{2}+\right. \\
& \left.+\|\boldsymbol{\beta}: \mathbf{e}(\widehat{\mathbf{u}})\|_{L^{2}\left(0, T ; L^{2}(\Omega)\right)}^{2}+\|\varrho\|_{L^{2}\left(0, T ; L^{2}\left(\Gamma_{m N}\right)\right)}^{2}\right)
\end{aligned}
$$


$\Xi_{p}$ is thus bounded and admits a subsequence still denoted $\Xi_{p}$ such that

$$
\Xi_{p} \rightarrow \Xi \text { in } L^{2}\left(0, T ; H_{t D}^{*}(\Omega)\right) .
$$

Now, spaces $\mathbf{V}_{p}\left(\Omega, \Gamma_{m D}\right)$ and $V_{p}\left(\Omega, \Gamma_{t D}\right)$ are dense, respectively, in $\mathbf{H}_{m D}^{1}(\Omega)$ and $H_{t D}^{1}(\Omega)$. By multiplying (2.20) and the equations of system (2.21) by a test function $\lambda \in \mathcal{D}(0, T)$, integrating in time, passing to the limit, taking into account (2.28)(2.29)-(2.30) and exploiting the arbitrariness of $\lambda$, we obtain

$$
\begin{gathered}
A(\overline{\mathcal{U}}(t), \mathcal{V})=\bar{L}(\mathcal{V}), \\
\forall \mathcal{V} \in \mathbf{H}_{m D}^{1}(\Omega) \times H_{e D}^{1}(\Omega) \times H_{g D}^{1}(\Omega) \times H_{t D}^{1}(\Omega), t \in(0, T)
\end{gathered}
$$

Again, from (2.28)-(2.29)-(2.30), we infer the regularity properties of the solution:

$$
\left\{\begin{array}{l}
\overline{\mathbf{u}} \in L^{2}\left(0, T ; \mathbf{H}_{m D}^{1}(\Omega)\right) \cap C^{0}\left([0, T] ; \mathbf{L}^{2}(\Omega)\right), \\
\partial_{t} \overline{\mathbf{u}} \in L^{2}\left(0, T ; \mathbf{L}^{2}(\Omega)\right), \\
\rho \partial_{t t} \overline{\mathbf{u}} \in L^{2}\left(0, T ; \mathbf{H}_{m D}^{*}(\Omega)\right) \\
\varphi \in L^{2}\left(0, T ; H_{e D}^{1}(\Omega)\right), \\
\zeta \in L^{2}\left(0, T ; H_{g D}^{1}(\Omega)\right), \\
\theta \in L^{2}\left(0, T ; H_{t D}^{1}(\Omega)\right), \\
c_{v} \dot{\theta}+\boldsymbol{\beta}: \partial_{t} \mathbf{e}(\overline{\mathbf{u}})-\mathbf{p} \cdot \nabla \dot{\varphi}-\mathbf{m} \cdot \nabla \dot{\zeta} \in L^{2}\left(0, T ; H_{t D}^{*}(\Omega)\right)
\end{array}\right.
$$

It remains to show that the weak limits $\overline{\mathbf{u}}, \partial_{t} \overline{\mathbf{u}}$ and $\theta$ satisfy the imposed initial conditions $\overline{\mathbf{u}}(0)=\overline{\mathbf{u}}_{0}, \partial_{t} \overline{\mathbf{u}}(0)=\overline{\mathbf{u}}_{1}$ and $\theta(0)=\theta_{0}$. As in [25], to prove that $\overline{\mathbf{u}}(0)=$ $\overline{\mathbf{u}}_{0}$, we choose $\lambda \in C^{1}([0, T])$ such that $\lambda(0)=1$ and $\lambda(T)=0$; an application of (2.28) yields

$$
\int_{0}^{T}\left(\partial_{t} \overline{\mathbf{u}}_{p}(t), \mathbf{v}\right) \lambda(t) \mathrm{d} t \rightarrow \int_{0}^{T}\left(\partial_{t} \overline{\mathbf{u}}(t), \mathbf{v}\right) \lambda(t) \mathrm{d} t, \quad \forall \mathbf{v} \in \mathbf{H}_{m D}^{1}(\Omega)
$$

upon integrating by parts, we get

$$
-\left(\overline{\mathbf{u}}_{p}(0), \mathbf{v}\right)-\int_{0}^{T}\left(\overline{\mathbf{u}}_{p}(t), \mathbf{v}\right) \lambda^{\prime}(t) \mathrm{d} t \rightarrow-(\overline{\mathbf{u}}(0), \mathbf{v})-\int_{0}^{T}(\overline{\mathbf{u}}(t), \mathbf{v}) \lambda^{\prime}(t) \mathrm{d} t .
$$

Now, since $\int_{0}^{T}\left(\overline{\mathbf{u}}_{p}(t), \mathbf{v}\right) \lambda^{\prime}(t) \mathrm{d} t \rightarrow \int_{0}^{T}(\overline{\mathbf{u}}(t), \mathbf{v}) \lambda^{\prime}(t) \mathrm{d} t$ by (2.28), we have

$$
\left(\overline{\mathbf{u}}_{p}(0), \mathbf{v}\right) \rightarrow(\overline{\mathbf{u}}(0), \mathbf{v}), \quad \forall \mathbf{v} \in \mathbf{H}_{m D}^{1}(\Omega),
$$

i.e., $\overline{\mathbf{u}}_{p}(0) \rightarrow \overline{\mathbf{u}}(0)$ in $\mathbf{L}^{2}(\Omega)$. By $(2.22)_{1}$ and the uniqueness of limit we get $\overline{\mathbf{u}}(0)=\overline{\mathbf{u}}_{0}$. Analogously we show that $\partial_{t} \overline{\mathbf{u}}(0)=\overline{\mathbf{u}}_{1}$ and $\theta(0)=\theta_{0}$.

Step 6. The solution $\overline{\mathcal{U}}(t)$ is unique. By contradiction, if there exist two solutions $\left(\overline{\mathbf{u}}^{*}, \varphi^{*}, \zeta^{*}, \theta^{*}\right)$ and $\left(\overline{\mathbf{u}}_{*}, \varphi_{*}, \zeta_{*}, \theta_{*}\right)$ of $(2.18)$, set $\mathbf{w}:=\overline{\mathbf{u}}^{*}-\overline{\mathbf{u}}_{*}, \psi:=\varphi^{*}-\varphi_{*}$, $\xi:=\zeta^{*}-\zeta_{*}, \eta:=\theta^{*}-\theta_{*}$, then $\mathbf{W}:=(\mathbf{w}, \psi, \xi, \eta)$, and consider the problem

$$
\left\{\begin{array}{l}
\rho \ddot{\mathbf{W}}-\operatorname{div} \boldsymbol{\sigma}(\mathbf{W})=\mathbf{0}, \\
\operatorname{div} \mathbf{D}(\mathbf{W})=0, \\
\operatorname{div} \mathbf{B}(\mathbf{W})=0, \\
\dot{s}(\mathbf{W})+\operatorname{div} \mathbf{q}(\eta)=0,
\end{array}\right.
$$


with vanishing initial and boundary conditions. Denote by $\mathcal{E}(\mathbf{W}(t))$ the energy associated with $\mathbf{W}$ at time $t$; then, by (2.11) we get

$$
\dot{\mathcal{E}}(\mathbf{W}(t)) \leq \dot{\mathcal{E}}(\mathbf{W}(t))+a_{\theta}(\eta(t), \eta(t))=0,
$$

whence, by integration, $\mathcal{E}(\mathbf{W}(t)) \leq \mathcal{E}(\mathbf{W}(0))=0$. This implies in turn that $a_{\theta}(\eta(t), \eta(t))=0$ and thus $\mathbf{W}=\mathbf{0}$.

\section{Another justification of the quasi-static assumption}

In this subsection, we outline a study of the limit as $\delta \rightarrow 0$ of the solution to problem (1.19)-(1.20)-(1.21). We start by rewriting the nondimensionalized system (2.2):

$$
\left\{\begin{array}{l}
\rho_{r} \ddot{\mathbf{u}}_{r}-\operatorname{div} \mathbf{C}_{r} \mathbf{e}\left(\mathbf{u}_{r}\right)+\chi \operatorname{div} \mathbf{P}_{r}^{T} \mathbf{E}_{r}+\kappa \operatorname{div} \mathbf{R}_{r}^{T} \mathbf{H}_{r}+\gamma \operatorname{div} \boldsymbol{\beta}_{r} \theta_{r}=\mathbf{f}_{r}, \\
\mathbf{X}_{r} \dot{\mathbf{E}}_{r}+\chi \mathbf{P}_{r} \mathbf{e}\left(\dot{\mathbf{u}}_{r}\right)+\alpha_{+} c_{0} \boldsymbol{\alpha}_{r} \dot{\mathbf{H}}_{r}+\varsigma \mathbf{p}_{r} \dot{\theta}_{r}-\delta^{-1} \nabla \times \mathbf{H}_{r}=-\mathbf{J}_{r}, \\
\mathbf{M}_{r} \dot{\mathbf{H}}_{r}+\kappa \mathbf{R}_{r} \mathbf{e}\left(\dot{\mathbf{u}}_{r}\right)+\alpha_{+} c_{0} \boldsymbol{\alpha}_{r} \dot{\mathbf{E}}_{r}+v \mathbf{m}_{r} \dot{\theta}_{r}+\delta^{-1} \nabla \times \mathbf{E}_{r}=\mathbf{0}, \\
c_{v r} \dot{\theta}_{r}+\gamma \boldsymbol{\beta}_{r}: \mathbf{e}\left(\dot{\mathbf{u}}_{r}\right)+\varsigma\left(\mathbf{p}_{r} \cdot \dot{\mathbf{E}}_{r}\right)+v\left(\mathbf{m}_{r} \cdot \dot{\mathbf{H}}_{r}\right)-\lambda \operatorname{div} \mathbf{K}_{r} \nabla \theta_{r}=r_{r},
\end{array}\right.
$$

where we have set ${ }^{\mathrm{q}}$

$$
\gamma:=\frac{\beta_{+}}{V_{+} \sqrt{\rho_{+} c_{v+}}} \simeq 30, \quad \lambda:=\frac{K_{+}}{V_{+} \mathcal{L} c_{v+} T_{0}} \simeq 1.6 \cdot 10^{-7} .
$$

We recall that all the equations hold for $x \in \widehat{\Omega}$ (we use an analogous notation for boundary partitions) and $t>0$, and that $x$ and $t$ are dimensionless. The boundary conditions are

$$
\left\{\begin{array} { l l } 
{ \boldsymbol { \sigma } _ { r } ( \widetilde { \mathcal { U } } _ { r } ) \mathbf { n } _ { r } = \mathbf { g } _ { r } } & { \text { on } \widehat { \Gamma } _ { m N } , } \\
{ - \mathbf { q } _ { r } ( \theta _ { r } ) \cdot \mathbf { n } _ { r } = \varrho _ { r } } & { \text { on } \widehat { \Gamma } _ { t N } , }
\end{array} \quad \left\{\begin{array}{ll}
\mathbf{u}_{r}=\mathbf{0} & \text { on } \widehat{\Gamma}_{m D} \\
\mathbf{E}_{r} \times \mathbf{n}_{r}=\mathbf{0} & \text { on } \widehat{\Gamma}_{e D} \\
\mathbf{H}_{r} \times \mathbf{n}_{r}=\mathbf{0} & \text { on } \widehat{\Gamma}_{g D} \\
\theta_{r}=0 & \text { on } \widehat{\Gamma}_{t D}
\end{array}\right.\right.
$$

where $\widetilde{\mathcal{U}}_{r}:=\left(\mathbf{u}_{r}, \mathbf{E}_{r}, \mathbf{H}_{r}, \theta_{r}\right), \mathbf{n}_{r}$ is the outer unit normal vector field over $\partial \widehat{\Omega}$, $\boldsymbol{\sigma}_{r}\left(\widetilde{\mathcal{U}}_{r}\right):=\mathbf{C}_{r} \mathbf{e}\left(\mathbf{u}_{r}\right)-\kappa \mathbf{R}_{r}^{T} \mathbf{H}_{r}-\chi \mathbf{P}_{r}^{T} \mathbf{E}_{r}-\gamma \boldsymbol{\beta}_{r} \theta_{r}$ and $\mathbf{q}_{r}\left(\theta_{r}\right):=-\mathbf{K}_{r} \nabla \theta_{r}$. Again, we omit boundary conditions on the normal components of the magnetic induction $\mathbf{B}_{r}$ and of the electric displacement $\mathbf{D}_{r}$ as they just impose compatilibility conditions on the choice of initial values $\mathbf{u}_{r}^{0}, \mathbf{E}_{r}^{0}, \mathbf{H}_{r}^{0}$ and $\theta_{r}^{0}$ and of the initial nondimensional electric charge density $\rho_{e_{r}}^{0}$.

A first hint that the limit solution of (3.1) as $\delta \rightarrow 0$ is such that $\nabla \times \mathbf{E}_{r}=\mathbf{0}$ and $\nabla \times \mathbf{H}_{r}=\mathbf{0}$ can be obtained by expressing the $L^{2}{ }_{-}$norm of $\nabla \times \mathbf{E}_{r}^{0}$ and of $\nabla \times \mathbf{H}_{r}^{0}$ in terms of the $L^{2}$-norm of $\nabla \times \mathbf{E}_{0}$ and of $\nabla \times \mathbf{H}_{0}$. Indeed, for any $x \in \Omega$, we have

$$
\mathbf{E}_{r}^{0}\left(\frac{x}{\mathcal{L}}\right)=\frac{1}{E^{*}} \mathbf{E}_{0}(x)=\frac{1}{V_{+}} \sqrt{\frac{\epsilon_{0}}{\rho_{+}}} \mathbf{E}_{0}(x)=\frac{\delta}{V_{+}^{2} \sqrt{\mu_{0} \rho_{+}}} \mathbf{E}_{0}(x),
$$

${ }^{\mathrm{q}}$ To calculate $\lambda$, we take $T_{0}=297 \mathrm{~K}$ as a reference temperature, as in [21]; for what concerns the thermal conductivity, we take the weighted average of the values provided in [27], since numerical values for the $\mathrm{BaTiO}_{3}-\mathrm{CoFe}_{2} \mathrm{O}_{4}$ composite with volume fraction 0.6 of $\mathrm{BaTiO}_{3}$ are not available in literature. As for the length scale, we take $\mathcal{L}=3 \mathrm{~cm}$. 
whence

$$
\nabla \times \mathbf{E}_{r}^{0}\left(\frac{x}{\mathcal{L}}\right)=\frac{\delta \mathcal{L}}{V_{+}^{2} \sqrt{\mu_{0} \rho_{+}}} \nabla \times \mathbf{E}_{0}(x) .
$$

Upon integrating the squared euclidean norm of both members of this equality, we find

$$
\begin{aligned}
\int_{\Omega}\left|\nabla \times \mathbf{E}_{r}^{0}\left(\frac{x}{\mathcal{L}}\right)\right|^{2} \mathrm{~d} \Omega & =\mathcal{L}^{3} \int_{\widehat{\Omega}}\left|\nabla \times \mathbf{E}_{r}^{0}(y)\right|^{2} \mathrm{~d} \widehat{\Omega}= \\
& =\mathcal{L}^{3}\left\|\nabla \times \mathbf{E}_{r}^{0}\right\|_{\mathbf{L}^{2}(\widehat{\Omega})}^{2}=\frac{\delta^{2} \mathcal{L}^{2}}{V_{+}^{4} \mu_{0} \rho_{+}}\left\|\nabla \times \mathbf{E}_{0}\right\|_{\mathbf{L}^{2}(\Omega)}^{2},
\end{aligned}
$$

that is to say,

$$
\left\|\nabla \times \mathbf{E}_{r}^{0}\right\|_{\mathbf{L}^{2}(\widehat{\Omega})}=\frac{\delta}{V_{+}^{2} \sqrt{\mu_{0} \rho_{+} \mathcal{L}}}\left\|\nabla \times \mathbf{E}_{0}\right\|_{\mathbf{L}^{2}(\Omega)} ;
$$

analogously, we obtain

$$
\left\|\nabla \times \mathbf{H}_{r}^{0}\right\|_{\mathbf{L}^{2}(\widehat{\Omega})}=\frac{\delta}{V_{+}^{2} \sqrt{\epsilon_{0} \rho_{+} \mathcal{L}}}\left\|\nabla \times \mathbf{H}_{0}\right\|_{\mathbf{L}^{2}(\Omega)} .
$$

The denominators appearing on the right-hand sides of these equalities are, of course, not dimensionless, and have the following numerical values:

$$
\begin{gathered}
V_{+}^{2} \sqrt{\mu_{0} \rho_{+} \mathcal{L}} \simeq 5.2 \cdot 10^{5} \mathrm{Vm}^{-1 / 2}=O\left(\delta^{-1}\right) \mathrm{V} \mathrm{m}^{-1 / 2}, \\
V_{+}^{2} \sqrt{\epsilon_{0} \rho_{+} \mathcal{L}} \simeq 1.38 \cdot 10^{3} \mathrm{~A} \mathrm{~m}^{-1 / 2}=O\left(\delta^{-3 / 5}\right) \mathrm{A} \mathrm{m}^{-1 / 2},
\end{gathered}
$$

therefore, unless $\left\|\nabla \times \mathbf{E}_{0}\right\|_{\mathbf{L}^{2}(\Omega)}=O\left(\delta^{-2}\right) \mathrm{Vm}^{-1 / 2}$ and $\left\|\nabla \times \mathbf{H}_{0}\right\|_{\mathbf{L}^{2}(\Omega)}=$ $O\left(\delta^{-8 / 5}\right) \mathrm{A} \mathrm{m}^{-1 / 2}$, which is never the case in applications, we have

$$
\lim _{\delta \rightarrow 0}\left\|\nabla \times \mathbf{E}_{r}^{0}\right\|_{\mathbf{L}^{2}(\widehat{\Omega})}=\lim _{\delta \rightarrow 0}\left\|\nabla \times \mathbf{H}_{r}^{0}\right\|_{\mathbf{L}^{2}(\widehat{\Omega})}=0 .
$$

\section{Conclusions}

We presented a mathematical model to describe linear magneto-electro-thermoelastic materials, as well as the proofs of the well-posedness for the problem formulated in its most general setting (dynamic) and for the quasi-static problem, based on the assumption that the electric and magnetic fields can be expressed as gradients of the correspondent potentials. We set forth a first validation of this hypothesis by carrying out a formal nondimensionalization procedure on the equations of the dynamic problem. A rigorous mathematical justification analogous to that set forth in [18] for a piezoelectric material needs further work, since the dynamic problem has been solved in the context of the Hille-Yosida theory, obtaining a smooth solution in time, whereas the solution of the quasi-static problem has been obtained in a weak form by the Faedo-Galerkin method. 
A problem that has already been addressed [5] is the deduction of a plate model for a magneto-electro-thermo-elastic sensor/actuator, based on the quasi-static assumption, by means of the asymptotic expansion method, taking into account another small parameter $\varepsilon$ tending to zero and characterizing the ratio of the plate thickness to the plate diameter. Another interesting extension for what concerns applications is the study of a laminated structure (plate-like or shell-like) including a thin magneto-electro-thermo-elastic layer; for the case of a piezoelectric layer see, e.g., [29] and [30].

Finally, in view of applications, a natural problem to be dealt with at a later stage is to come up with efficient numerical methods to perform simulations of the equations involved in the problem.

\section{Appendix}

\section{Numerical Values of the Materials Constants}

The table below lists numerical values of the material properties for a $\mathrm{BaTiO}_{3}-\mathrm{CoFe}_{2} \mathrm{O}_{4}$ composite with 0.6 volume fraction of barium titanate. We took Table 1 in [22] as a reference. The two diagonal components $M_{11}$ and $M_{22}$ of the magnetic permeability tensor assume negative values in [22]. This would contradict our hypothesis (1.12), but it is actually a widespread error in literature, based on the incorrect determination of the sign of these coefficients for the pure cobalt ferrite $\mathrm{CoFeO}_{2}$, as pointed out in [14], [21], [20] and [32]. Therefore, we corrected the values of these two components by changing their sign.

Table 1. Material properties of a magneto-electro-thermo-elastic $\mathrm{BaTiO}_{3}-\mathrm{CoFe}_{2} \mathrm{O}_{4}$ composite with 0.6 volume fraction of $\mathrm{BaTiO}_{3}$.

\begin{tabular}{|c|c|c|c|}
\hline \multirow{3}{*}{$\begin{array}{c}\text { Elastic moduli } \\
C_{1111}=C_{2222}(\mathrm{GPa}) \\
C_{1122}(\mathrm{GPa})\end{array}$} & \multirow{4}{*}{$\begin{array}{l}200 \\
110 \\
110\end{array}$} & \multirow{3}{*}{$\begin{array}{c}\text { Magnetic permeabilities } \\
M_{11}=M_{22}\left(10^{-4} \mathrm{~N} \mathrm{~s}^{2} / \mathrm{C}^{2}\right) \\
M_{33}\left(10^{-4} \mathrm{~N} \mathrm{~s}^{2} / \mathrm{C}^{2}\right)\end{array}$} & \multirow{3}{*}{$\begin{array}{c}1.5 \\
0.75\end{array}$} \\
\hline & & & \\
\hline & & & \\
\hline$C_{1133}=C_{2233}(\mathrm{GPa})$ & & Piezomagnetic constants & \\
\hline$C_{3333}(\mathrm{GPa})$ & 190 & $R_{311}=R_{322}(\mathrm{~N} / \mathrm{Am})$ & 200 \\
\hline$C_{2323}=C_{3131}(\mathrm{GPa})$ & 45 & $R_{333}(\mathrm{~N} / \mathrm{Am})$ & 260 \\
\hline$C_{1212}(\mathrm{GPa})$ & 45 & $R_{113}(\mathrm{~N} / \mathrm{Am})$ & 180 \\
\hline Piezoelectric constants & & Magnetoelectric constants & \\
\hline$P_{311}=P_{322}\left(\mathrm{C} / \mathrm{m}^{2}\right)$ & -3.5 & $\alpha_{11}=\alpha_{22}\left(10^{-12} \mathrm{Ns} / \mathrm{VC}\right)$ & 6 \\
\hline$P_{333}\left(\mathrm{C} / \mathrm{m}^{2}\right)$ & 11 & $\alpha_{33}\left(10^{-12} \mathrm{~N} \mathrm{~s} / \mathrm{V} \mathrm{C}\right)$ & 2500 \\
\hline \multirow{3}{*}{$\begin{array}{c}\text { Dielectric permittivities } \\
X_{11}=X_{22}\left(10^{-9} \mathrm{C}^{2} / \mathrm{N} \mathrm{m}^{2}\right) \\
X_{33}\left(10^{-9} \mathrm{C}^{2} / \mathrm{N} \mathrm{m}^{2}\right)\end{array}$} & \multirow{3}{*}{$\begin{array}{l}0.9 \\
7.5\end{array}$} & Pyroelectric constants & \multirow[b]{2}{*}{-12.4} \\
\hline & & $p_{2}\left(10^{-5} \mathrm{C} / \mathrm{m}^{2} \mathrm{~K}\right)$ & \\
\hline & & \multirow{2}{*}{$\begin{array}{l}\text { Pyromagnetic constants } \\
m_{2}\left(10^{-3} \mathrm{~N} / \mathrm{Am} \mathrm{K}\right)\end{array}$} & \multirow[b]{2}{*}{5.92} \\
\hline \multirow{2}{*}{$\begin{array}{c}\text { Thermal stresses } \\
\beta_{11}=\beta_{22}\left(10^{6} \mathrm{~N} / \mathrm{K} \mathrm{m}^{2}\right) \\
\beta_{33}\left(10^{6} \mathrm{~N} / \mathrm{K} \mathrm{m}^{2}\right)\end{array}$} & \multirow[b]{2}{*}{$\begin{array}{l}4.86 \\
4.32\end{array}$} & & \\
\hline & & $\begin{array}{l}\text { Density } \\
\rho\left(\mathrm{kg} / \mathrm{m}^{3}\right)\end{array}$ & 5600 \\
\hline $\begin{array}{c}\text { Thermal conductivities } \\
K_{33}(\mathrm{~W} / \mathrm{m} \mathrm{K})\end{array}$ & 2.85 & $\begin{array}{l}\text { Calorific capacity } \\
c_{v}\left(\mathrm{~J} / \mathrm{m}^{3} \mathrm{~K}^{2}\right)\end{array}$ & 325 \\
\hline
\end{tabular}

In our notation, $\boldsymbol{\beta}$ denotes the thermal stress tensor, whereas in [22] it denotes the 
thermal expansion tensor. For $\gamma$ the thermal expansion tensor and $\mathbf{C}$ the elasticity tensor, the thermal stress tensor $\boldsymbol{\beta}$ is given by the relation $\boldsymbol{\beta}=\mathbf{C} \boldsymbol{\gamma}$, which we used to infer the values of the thermal stress components. Also, since no constitutive assumption concerning entropy, as well as heat influx, is made in [22], numerical values for the calorific capacity and the thermal conductivities are unavailable in [22]. However, an estimate of these values can be retrieved, respectively, from [21] and [27].

\section{The Hille-Yosida Theorem}

The statement of the Hille-Yosida theorem for a linear non-homogeneous differential equation employed in the paper is the following (see [6]).

Theorem 3.1. Let $\mathbb{H}$ be a Hilbert space, $\mathcal{A}: D(\mathcal{A}) \subset \mathbb{H} \rightarrow \mathbb{H}$ a linear operator with domain $D(\mathcal{A})$, a linear subspace of $\mathbb{H}$. Let $\mathcal{A}$ be a maximal monotone operator. Then, for any $U_{0} \in D(\mathcal{A})$ and $F \in C^{1}([0, T] ; \mathbb{H})$, the problem

$$
\left\{\begin{array}{l}
\frac{\mathrm{d} U}{\mathrm{~d} t}+\mathcal{A} U=F, \quad t>0, \\
U(0)=U_{0}
\end{array}\right.
$$

admits a unique strong solution

$$
U \in C^{1}([0, T] ; \mathbb{H}) \cap C^{0}([0, T] ; D(\mathcal{A})) .
$$

\section{Acknowledgements}

This work has been supported by the French Agence Nationale de la Recherche (ANR) under grant ARAMIS (Projet «Blanc», N. ANR 12 BS01-0021) (Analysis of Robust Asymptotic Methods In numerical Simulation in mechanics).

\section{References}

1. J. Aboudi, Micromechanical analysis of fully coupled electro-magneto-thermo-elastic multiphase composites, Smart Mater. Struct. 10 (2001), 867-877.

2. S. N. Ahmad, C. S. Upadhyay, C. Venkatesan, Electro-thermo-elastic formulation for the analysis of smart structures, J. Smart Materials and Structures, 15 No 2, (2006), 401-416.

3. M. Aouadi, The Generalized Theory of Thermo-Magnetoelectroelasticity, Technische Mechanik, Band 27, Heft 2 (2007), 133-146.

4. M. H. Babaei, Z. T. Chen, The transient coupled thermo-piezoelectric response of a functionally graded piezoelectric hollow cylinder to dynamic loadings, Proc. R. Soc. A (2010) 466, 1077-1091.

5. F. Bonaldi, G. Geymonat, F. Krasucki, M. Serpilli, Temperature influence on smart structures: a first approach, Proceedings of the World Congress on Computational Mechanics, ISBN/ISSN: 9788494284472 (2014).

6. H. Brezis, Functional Analysis, Sobolev Spaces and Partial Differential Equations, Springer, New York (2011). 
7. A. Buffa, M. Costabel, D. Sheen, On traces for $\mathbf{H}(\operatorname{curl}, \Omega)$ in Lipschitz domains, J. Math. Anal. Appl. 276 (2002) 845-867.

8. G. P. Carman, M. Mitrovic, Nonlinear Constitutive Relations for Magnetostrictive Materials with Applications to 1-D Problems, Journal of Intelligent Material Systems and Structures (1995), 673-683.

9. K. S. Challagulla, A. V. Georgiades, Micromechanical analysis of magneto-electrothermo-elastic composite materials with applications to multilayered structures, International Journal of Engineering Science 49 (2011), 85-104.

10. D. S. Chandrasekharaiah, A generalized linear thermoelasticity theory for piezoelectric media, Acta Mechanica 71 (1988), 39-49.

11. B. D. Coleman, W. Noll, The thermodynamics of elastic materials with heat conduction and viscosity, Arch. Rational Mech. Anal. 13, (1963), 167-178.

12. S. Giordano, Y. Dusch, N. Tiercelin, P. Pernod, V. Preobrazhensky, Combined nanomechanical and nanomagnetic analysis of magnetoelectric memories, Physical Review B 85 (2012), 155321.

13. S. Giordano, Y. Dusch, N. Tiercelin, P. Pernod, V. Preobrazhensky, Thermal effects in magnetoelectric memories with stress-mediated switching, J. Phys. D: Appl. Phys. 46 (2013), 325002.

14. S. Giordano, M. Goueygou, N. Tiercelin, A. Talbi, P. Pernod, V. Preobrazhensky, Magneto-electro-elastic effective properties of multilayered artificial multiferroics with arbitrary lamination direction, International Journal of Engineering Science 78 (2014), 134-153.

15. V. Girault, P. A. Raviart, Finite Element Methods for Navier-Stokes Equations, Springer Verlag, Berlin (1986).

16. D. A. Hadjiloizi, A. V. Georgiades, A. L. Kalamkarov, S. Jothi, Micromechanical modeling of piezo-magneto-thermo-elastic composite structures: Part I - Theory, European Journal of Mechanics A/Solids 39 (2013), 298-312.

17. D. A. Hadjiloizi, A. V. Georgiades, A. L. Kalamkarov, S. Jothi, Micromechanical modeling of piezo-magneto-thermo-elastic composite structures: Part II - Applications, European Journal of Mechanics A/Solids 39 (2013), 313-327.

18. S. Imperiale, P. Joly, Mathematical and numerical modelling of piezoelectric sensors, ESAIM: Mathematical Modelling and Numerical Analysis (2012), 875-909.

19. S. Imperiale, Etude mathématique et numérique de capteurs piézoélectriques, $\mathrm{PhD}$ Thesis, Université Paris Dauphine (2012).

20. J. Y. Kim, Micromechanical analysis of effective properties of magneto-electro-thermoelastic multilayer composites, International Journal of Engineering Science 49 (2011), 1001-1018.

21. J. Y. Kim, Z. Li, A. Baltazar, Pyroelectric and pyromagnetic coefficients of functionally graded multilayered multiferroic composites, Acta Mech 223 (2012), 849-860.

22. P. Kondaiah, K. Shankar, N. Ganesan, Pyroelectric and pyromagnetic effects on behavior of magneto-electro-elastic plate, Coupled Systems Mechanics, Vol. 2, No. 1 (2013), 1-22.

23. P. Kondaiah, K. Shankar, N. Ganesan, Pyroeffects on Multiphase Magneto-ElectroElastic Sensor Patch Bonded on Mild Steel Plate, Journal of Smart Sensing and Intelligent Systems, Vol 7(3) (2014), 1134-1155.

24. J. L. Lions, Equations différentielles opérationnelles et problèmes aux limites, Springer Verlag, Berlin (1961).

25. B. Miara, J. S. Suárez, Asymptotic pyroelectricity and pyroelasticity in thermopiezoelectric plates, Asymptotic Analysis 81 (2013), 211-250.

26. C. W. Nan, M. I. Bichurin, S. Dong, D. Viehland, G. Srinivasan, Multiferroic magne- 
toelectric composites: Historical perspective, status, and future directions, Journal of Applied Physics 103 (2008).

27. Y. Ootao, Y. Tanigawa, Transient analysis of multilayered magneto-electrothermoelastic strip due to nonuniform heat supply, Composite Structures 68 (2005), 471-480.

28. J. Sanchez Hubert, E. Sanchez Palencia, Vibration and Coupling of Continuous Systems. Asymptotic Methods, Springer-Verlag, Berlin Heidelberg (1989).

29. M. Serpilli, An asymptotic model of a multimaterial with a thin piezoeletric interphase, C. R. Mécanique, Vol. 342, 258-262 (2014).

30. M. Serpilli, Asymptotic analysis of a multimaterial with a thin piezoelectric interphase, Meccanica, 1641-1652 (2014).

31. J.M.S. Suárez, Modelling of thermopiezoelectric phenomenon: asymptotic analysis and numerical simulation, PhD Thesis, National Laboratory of Scientific Computation, Brazil, (2011) (in Portuguese).

32. K. H. Sun, Y. Y. Kim, Layout design optimization for magneto-electro-elastic laminate composites for maximized energy conversion under mechanical loading, Smart Materials and Structures 19 (2010).

33. M. Sunar, A. Z. Al-Garni, M. H. Ali, R. Kahraman, Finite Element Modeling of Thermopiezomagnetic Smart Structures, AIAA Journal Vol. 40, No. 9 (2002).

34. T. Weller, C. Licht, Modeling of linearly electromagneto-elastic thin plates, C. R. Mécanique 335, (2007), 201-206. 